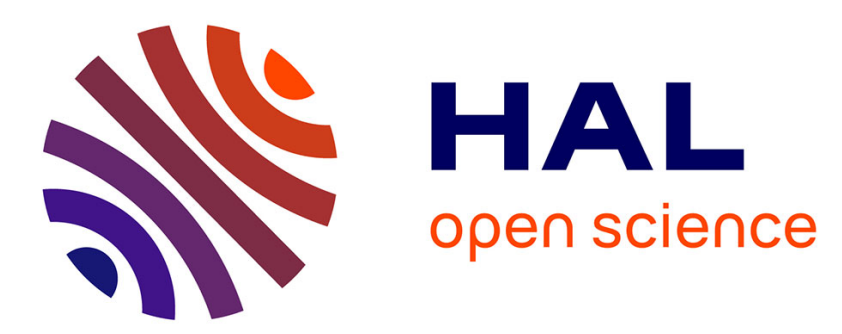

\title{
A survey on driving prediction techniques for predictive energy management of plug-in hybrid electric vehicles
}

\author{
Yang Zhou, Alexandre Ravey, Marie-Cécile Péra
}

\section{To cite this version:}

Yang Zhou, Alexandre Ravey, Marie-Cécile Péra. A survey on driving prediction techniques for predictive energy management of plug-in hybrid electric vehicles. Journal of Power Sources, 2019, 412, pp.480 - 495. hal-02130788

\section{HAL Id: hal-02130788 \\ https://hal.science/hal-02130788}

Submitted on 16 May 2019

HAL is a multi-disciplinary open access archive for the deposit and dissemination of scientific research documents, whether they are published or not. The documents may come from teaching and research institutions in France or abroad, or from public or private research centers.
L'archive ouverte pluridisciplinaire HAL, est destinée au dépôt et à la diffusion de documents scientifiques de niveau recherche, publiés ou non, émanant des établissements d'enseignement et de recherche français ou étrangers, des laboratoires publics ou privés. 


\title{
A Survey on Driving Prediction Techniques for Predictive Energy Management of Plug-in Hybrid Electric Vehicles
}

\author{
Yang Zhou ${ }^{1}$, Alexandre Ravey ${ }^{1}$ and Marie-Cécile Péra ${ }^{1}$
}

Abstract - Driving prediction techniques (DPTs) are used to forecast the distributions of various future driving conditions (FDC), like velocity, acceleration, driver behaviors etc. and the quality of prediction results has great impacts on the performance of corresponding predictive energy management strategies (PEMSs), e.g., fuel economy (FE), lifetime of battery etc. This survey presents a comprehensive study on existing DPTs. Firstly, a review on prediction objectives and major types of prediction algorithms are presented. Then a comparative study on various prediction approaches is carried out and suitable application scenarios for each approach are provided according to their characteristics. Moreover, prediction accuracy-affecting factors are analyzed and corresponding approaches for dealing with mispredictions are discussed in detail. Finally, the bottlenecks of current researches and future developing trends of DPTs are given. In general, this paper not only gives a comprehensive analysis and review of existing DPTs but also indicates suitable application scenarios for each prediction algorithm and summarizes potential approaches for handling the prediction inaccuracies, which will help prospective designers to select proper DPTs according to different applications and contribute to the further performance enhancements of PEMSs for hybrid electric vehicles (HEVs) and plug-in hybrid electric vehicles (PHEVs).
Keywords - Driving prediction techniques, Predictive energy management strategies, prediction accuracy, HEV, PHEV.

\section{INTRODUCTION}

In order to cope with global concentrating issues like energy crisis, air pollutions and health problems brought by conventional vehicles, electric propulsion systems as the secondary energy sources are added into the conventional internal combustion engines (ICE) based powertrain systems to form HEVs or PHEVs [1]. In these hybrid powertrains, the secondary energy sources can assist ICEs to always run in the high efficiency area and improve the dynamic performances of vehicles. Consequently, benefiting from such powertrain configurations, dependences on fossil fuels is reduced and thus less exhausted gases will be emitted, which will lead to cleaner energy transportations. However, how to establish an efficient and effective PEMSs for HEVs/PHEVs to both provide sufficient energy according to changeable external power requirements and achieve best FE is still a not-well-resolved issue [2].

yang.zhou@utbm.fr; alexandre.ravey@utbm.fr; marie-cecile.pera@univfcomte.fr).
${ }^{1}$ Y. Zhou, A. Ravey and MC. Péra are with FEMTO-ST (UMR CNRS 6174), FCLAB (FR CNRS 3539), Energy Department, Univ. Bourgogne FrancheComte, UTBM, Rue Thierry Mieg, F-90010 Belfort Cedex, France. (E-mail: 
Nomenclature

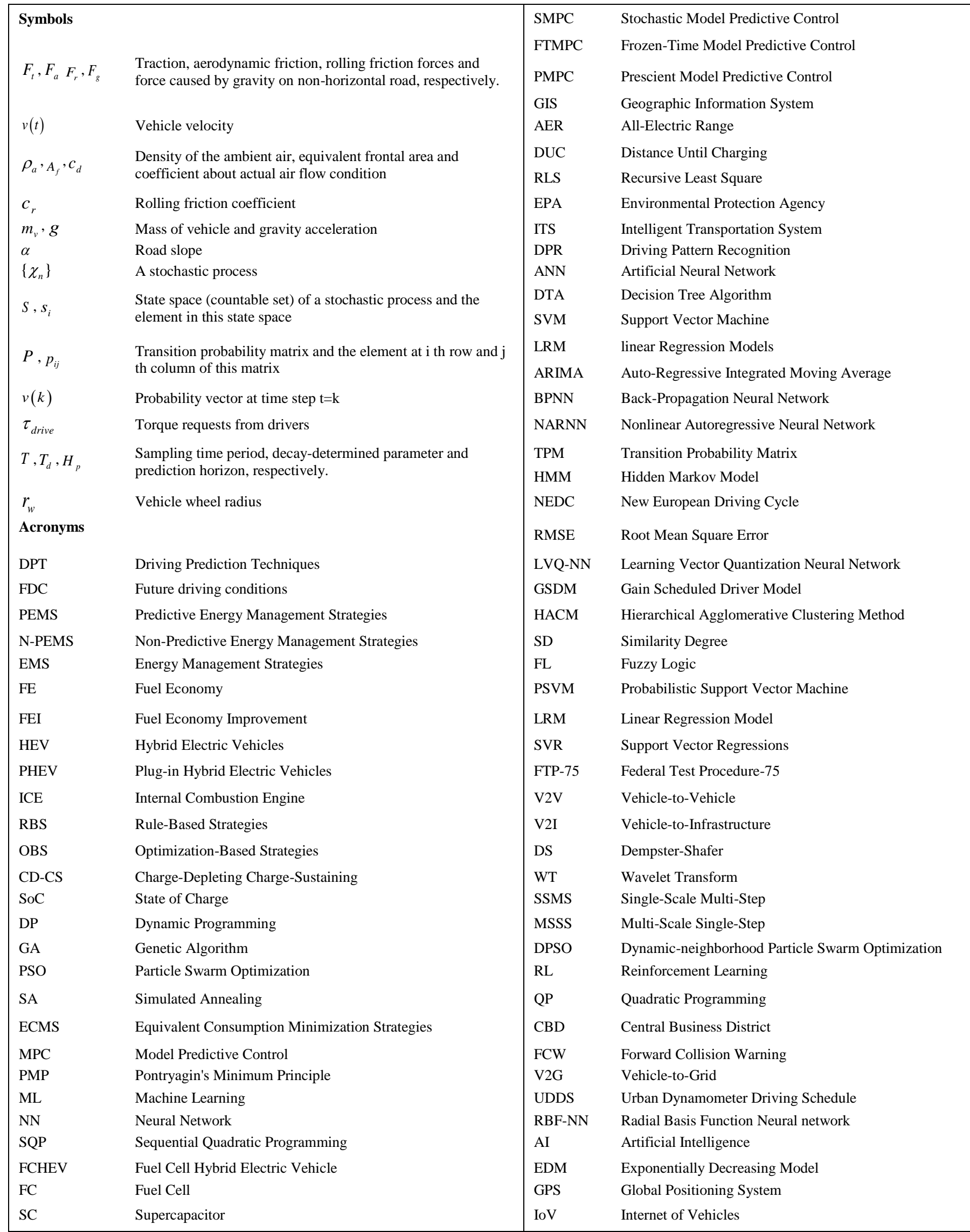


Based on previous researches, energy management strategies (EMSs) for HEVs/PHEVs can be classified into two main parts: Rule-based strategies (RBS) and Optimization-based strategies (OBS) [1], [3]-[5], where Charge-Depleting and ChargeSustaining (CD-CS) control strategy can be regarded as a special type of RBS for PHEVs. Attempts have been made by many researchers for establishing EMSs based on these methods. For example, as a representative RBS approach, the well-tuned CD-CS strategy based EMSs for PHEVs were widely used due to its simplicity and computational efficiency, which made the battery state of charge (SoC), an indicator of remaining electric charge in battery related to the nominal battery capacity [6], depleted at the beginning of trips and then maintained within tolerable boundaries when its value reached preset lower limits [7]-[11]. It is a simple-to-implement way of distributing the power flow within the hybrid powertrain system. However, it has shown sub-optimal [8], [12] or even non-optimal [13] performances on FE and corresponding electric system efficiency drop during intensive CD mode [9].

Compared with RBS approaches, OBSs have shown their advantages in improving FE by minimizing cost functions within various optimization algorithms, including dynamic programming (DP), genetic algorithm (GA), particle swarm optimization (PSO), simulated annealing (SA), etc. As a frequently-utilized OBS approach, dynamic programming (DP) is exploited by many researchers because it can provide with global optimal solutions on FE [14], [15] during entire trips. However, two facts prevent this method to be used for real time application: 1) large computational burdens (for the entire trip); 2) requirement of fully-previewed knowledge on future trip
[16]-[21], including both future road slope information and velocity profiles, which made them attractive but only in offline design processes or as benchmarks for evaluating other EMSs. Alternatively, many researchers have switched their research focuses to online OBSs, including equivalent consumption minimization strategies (ECMS), model predictive control (MPC), pontryagin's minimum principle (PMP), machine learning (ML), neural network (NN) etc. Due to its reasonable computation burdens and no requirements of previewed knowledge, ECMS transformed original global optimization problems into instantaneous ones, which gave optimal solutions to distribute instantaneous power demand among different energy sources at each step by minimizing objective functions. Although computation burdens of ECMS were greatly reduced compared to offline OBSs (e.g. DP-based EMSs), corresponding improvements on FE could be compromised. For example, $\mathrm{Li}$ et al. proposed a sequential quadratic programming (SQP) based ECMS for fuel cell hybrid electric vehicle (FCHEV) [22]. By implementing three different penalty functions, the proposed real-time controller could adjust the dynamic behaviors of fuel cell (FC), battery and supercapacitor (SC) according to external power demands. At the same time, it could also maintain FC operating in high efficiency zone with smoothed output current profiles. From corresponding experimental results, it was clear that the proposed EMS could be operated in real-time and did not require any future driving information. However, only $2.16 \%$ fuel economy improvement (FEI) was achieved compared with a benchmark RBS.

In order to seek larger FEI, researchers were eager to find an advanced control framework to combine the short-term 
prediction results with online OBSs. MPC is such an ideal predictive control framework in automotive industries whose main benefits lie in strong ability of handling multivariable and constraints in optimal control problems. Generally, the FE performance of MPC based strategies was considered as a tradeoff between DP and ECMS [3]. For example, authors in [23] compared the performances of DP, MPC and ECMS based EMSs, whose fuel consumption on Urban Dynamometer Driving Schedule (UDDS) driving cycle were $216.39 \mathrm{~g}, 228.51 \mathrm{~g}$ and $242.40 \mathrm{~g}$, respectively. It should be noted that a Radial Basis Function NN (RBF-NN) velocity predictor was embedded into MPC control framework to provide with short-term velocity forecasted profiles in each online rolling optimization part.

From the results proposed in [23], it was clear that, combined with velocity prediction results, MPC-based controller could achieve $6.4 \%$ more FEI than ECMS-based one, which did not use predicted knowledge. Consequently, such potential of FEI brought by short-term predictors made DPTs widely concerned by researchers especially in HEV/PHEV energy management field. Despite different ways of embedding short-term FDC predictors into online OBS controllers, this novel type of EMS, PEMS, has become an effective approach to manage power flows among different energy sources (e.g. ICE, FC and battery etc.) to seek further FEI especially for PHEVs. Generally speaking, benefiting from the future trajectories distribution of vehicle power demand in a finite horizon, PEMSs can actively make some adjustments of energy allocation in advance rather than passive adjustments made by conventional EMSs according to series of predefined rules, engine efficiency maps or instantaneous power demand.
In order to study the influences brought to traditional EMSs by various DPTs, a novel classification of EMSs for HEVs/PHEVs is proposed considering whether or not they were associated with results of FDCs prediction. The block diagram of this novel classification is shown in Fig. 1. PEMSs can be further sub-classified into three categories based on the levels of future driving information used when making predictions. "Full knowledge" based PEMSs referred to the situation where predictions were based on fully previewed (100\%) knowledge for a given horizon whereas "Zero knowledge" based PEMSs were based on non-previewed knowledge (0\%) [18]. Moreover, the major difference between "Zero knowledge" PEMSs and non-predictive EMSs (NPEMS) is that the latter depends not on any predicted knowledge but only on deterministic rules, human intuitions, expert experience etc.

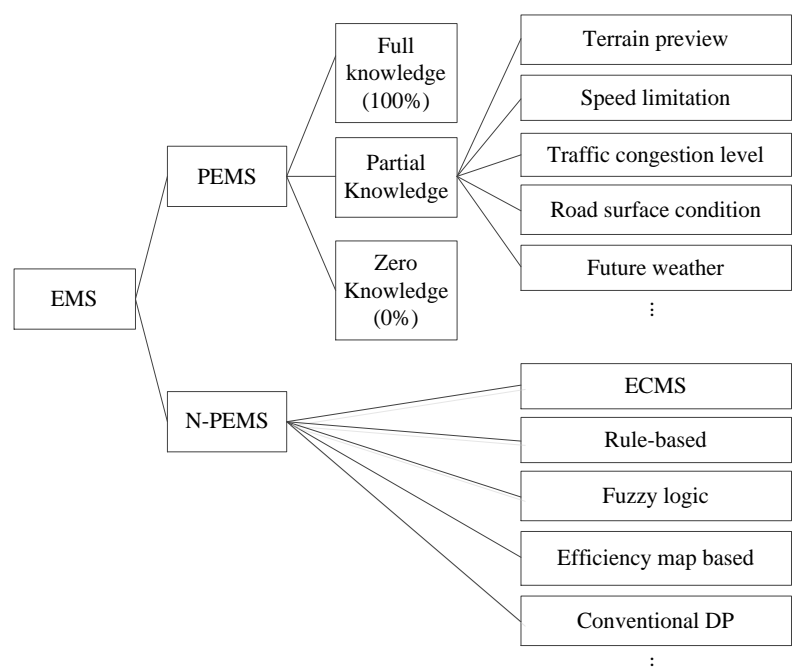

Fig. 1. Block diagram of novel classification of EMSs

Although there are many previous studies about various PEMSs for HEVs/PHEVs, there are little researches that focused on corresponding DPTs. Therefore, the motivation of this paper is to conduct a survey about various DPTs to help prospective designers for establishing corresponding PEMSs. 
The novelty of this paper lies in 1) analysis of several prediction objectives and corresponding reasons why they should be accurately predicted; 2) comparative study on existing approaches used for predicting FDCs; 3) discussions about proper application scenarios for each prediction approach, prediction accuracy-affecting factors and possible ways of improving prediction accuracy and robustness; 4) analysis about missing points in current researches and future trends of DPTs, which includes many original insights and comparisons to make this paper more than simple review on DPTs.

The remainder of the layout of this article is described as follows. In Section 2, a comprehensive review of DPTs is conducted, including several prediction objectives and existing FDCs prediction methods. In Section 3, a comparative study of their benefits and drawbacks is conducted and appropriate application scenarios for each approach are proposed. Then approaches for improving the performance of FCDs prediction methods are discussed. Moreover, the currently unsolved issues about prediction techniques and future developing trends of DPTs are given in Section 4. The conclusions are summarized in Section 5. Besides, all symbols and acronyms in this article could be found in Nomenclature Section.

\section{REVIEWS ON DRIVING PREDICTION TECHNIQUES}

The arrangement of section 2 is as follows: in section 2.1, several driving prediction objectives and corresponding reasons why these physical quantities are selected to be predicted are given in detail. Moreover, specific approaches, including Artificial Intelligence (AI) based approaches, Markov based approaches, Exponentially Decreasing Model (EDM) based approaches, telematics technique based approaches and other approaches, are discussed staring from section 2.2 to section 2.6, respectively. Finally, all types of prediction approaches and corresponding literatures are summarized at the end of this section.

\subsection{Driving prediction objectives}

In this sub-section, several driving prediction objectives, including velocity, acceleration, power demand, SoC reference trajectory, driving pattern, drivers' driving style, are introduced and the importance of providing reasonable prediction results on these objectives is analyzed in detail.

\subsubsection{Velocity, acceleration and power demand}

As it is stated in [6], the traction force and power demand of a road vehicle can be denoted as

$$
\begin{aligned}
& F_{t}(t)=m_{v} \frac{d}{d t} v(t)+F_{a}(t)+F_{r}(t)+F_{g}(t) \\
& =m_{v} \frac{d}{d t} v(t)+\frac{1}{2} \rho_{A} A_{f} c_{d} v(t)^{2}+c_{r} m_{v} g \cos (\alpha)+m_{v} g \sin (\alpha) \\
& P_{t}(t)=F_{t}(t) \cdot v(t)
\end{aligned}
$$

Where $F_{t}(t)$ is traction force, $F_{a}(t)$ aerodynamic friction, $F_{r}(t)$ the rolling friction, $F_{g}(t)$ the force caused by gravity when driving on non-horizontal roads, $v(t)$ is the vehicle speed, $\rho_{a}$ the density of the ambient air, $A_{f}$ the equivalent frontal area, $c_{d}$ the coefficient that model the actual air flow conditions, $c_{r}$ the rolling friction coefficient, $m_{v}$ the vehicle mass, $g$ the gravity acceleration and $\alpha$ the rode slope.

According to (1) and (2), it is clear that power demand from traction part of vehicles within prediction horizon are available given future information about vehicle velocity, acceleration and road slope. With the help of widely-equipped on-board 
GPS and Navigation systems, road slope information can be previewed when the desired origin-to-destination path is selected. However, vehicles velocities and accelerations are impossible to be previewed accurately within current transportation infrastructures due to different drivers' behaviors and uncertainties of real world driving conditions (e.g. traffic light distribution, unexpected movements of surrounding vehicles, etc.).

For these reasons, researchers are eager to find reasonable ways of predicting vehicle velocities, accelerations and power demands in the future. In previous researches [24], A NN-based future vehicle load estimator was established to optimize the control strategies on the supervisory level within prediction horizon, whose effectiveness was testified by simulations. Even though the quality of prediction along the entire trip cannot be guaranteed, it is verified that accessibility of high-accuracy predicted results in the near future can provide with considerable amount of fuel consumption saving. For example, in [23], PEMS based on MPC framework associated with RBFNN prediction model with 10 s prediction horizon consumed 228.51g of fuel, slightly more than that of DP benchmark, 216.39g, and less than that of ECMS (without prediction) based EMS strategy, 242.40g. Moreover, Park, J et al. proposed an intelligent vehicle power management strategy based on predictions of drive conditions (road type and traffic congestion level) and driving trends [25]. Compared to default controller embedded in PSAT software, the proposed intelligent power controller has achieved $9.39 \%, 11.16 \%$ and $5.35 \%$ fuel economy improvements (FEI) under three different driving cycles, which were close to those of DP based benchmark,
$13.84 \%, 13.79 \%$ and $15.28 \%$, respectively. Similarly, in [26], [27], authors proved that PEMSs based on Stochastic MPC (SMPC) associated with predicted power requests in short term showed fuel economy (FE) superiority than that of Frozen-time MPC (FTMPC), which is assumed that the future power demand hold as a constant value within prediction horizon. In addition, its performance is close to that of Prescient MPC (PMPC), which is assumed that the power request profile along entire trip is known in advance. Consequently, it is very necessary to provide with accurate prediction results of vehicle velocity, acceleration or power demand in the short term for larger FEI of proposed PEMSs.

\subsubsection{Battery SoC reference trajectory}

Battery SoC reference trajectories were pre-designed curves, which contained information about how to use energy stored in battery packs along specific trips. In EMSs for HEVs, basic principle of battery depletion strategy was to maintain battery SoC fluctuating within narrow ranges (e.g. 0.6-0.8), where final values of battery SoC were always expected to remain the same as its initial values. On the contrary, benefiting from larger capacity of on-board battery packs and additional plug-in property, designers expected to fully deplete battery energy of PHEVs at end of trips and then recharge them by external lowcost power grids. In that case, there will be more chances for saving primary energy sources (e.g. gasoline, diesel etc.) and limiting the emission of greenhouse gases.

Therefore, how to design such battery SoC reference curves became one of the researches focuses in recent years. At first, CD-CS strategy was widely investigated by researchers [12]. Although CD-CS strategy was a robust approach with less 
computation burdens, it has been testified that, compared with DP-based strategy, $22.17 \%$ more fuel was consumed by CD-CS strategy when real trip length was larger than all-electric range (AER) of PHEVs [4].

For these reasons, researchers were eager to pre-plan battery SoC reference values based on previewed terrain information and real-time traffic situations to guide vehicles' future energy allocation. Several efforts have been made by previous researches. For example, authors in [28] proposed a pattern recognition approach to estimate distance until charging (DUC) of PHEVs by several Recursive Least Square (RLS) based estimators. In that case, reference values of battery SoC within the range of each estimated DUC were designed to decrease linearly to its lower limits at the end of trip. Corresponding PEMS based on generated $\mathrm{SoC}$ references has shown an average of $1.82 \%$ FEI compared to CD-CS strategy. In [29], a bunch of optimized SoC reference trajectories under different driving cycles were used to train a neuro-fuzzy system, which was used to generate the near-optimal SoC reference trajectories for corresponding PEMS. General drawbacks of data-driven based SoC reference generation approaches in [28], [29] were 1) building training database containing sufficient driving conditions could be very time-consuming; 2) prediction accuracy could be highly affected if real driving conditions differed dramatically from those stored in training database. For these reasons, optimization-based SoC references generation approaches were explored by researchers. For example, authors in [13] proposed a SoC reference generation approach by solving an optimal problem online based on real-time traffic flow data and simplified PHEV powertrain model. Battery SoC trajectories obtained in the supervisory level were imported into lower level MPC controller as terminal SoC references. Simulation results showed that the proposed traffic dataenabled PEMS with predicted SoC references could achieve 94\%-96\% fuel optimality of DP-based benchmark. However, the requirement of real-time updating traffic data and computational burden with double optimization processes made it hard to use for real-time applications. Consequently, reasonable approaches for pre-planning SoC references trajectories are necessary to be further investigated.

\subsubsection{Driving patterns}

Driving pattern is a comprehensive description of the combination of road environment and the state of vehicles [30]. Congested urban roads, flowing urban roads, subway and highway are four typical driving patterns defined by Environmental Protection Agency (EPA) [31]. Strong uncertainties in realistic driving environments, such as random distribution of traffic lights, unexpected movements of pedestrians, made it very hard to obtain accurate predicted results on FDCs even though with previewed information on route and traffic from telematics systems. Compared to obtaining precise estimated values of corresponding physical quantities (e.g. velocity or power demand profiles etc.), vehicles' future driving patterns were much easier to be forecasted under the assumption that they will remain unchanged for a period of time. Moreover, future driving pattern information turned out to be helpful in improving the FE performances on corresponding PEMSs [32]. Consequently, many researchers turned their research focus from driving cycle prediction to driving pattern recognition (DPR) techniques. 
The basic principle of DPR is to sample and extract feature parameters from a period of past driving profiles. Then the welltrained classifiers are used to recognize the current driving patterns assuming that past driving patterns will remain unchanged in the short future [1]. Currently, several challenging issues exist for this technique: 1) the conflicts between recognition accuracy and computation burdens (e.g. number of parameters to characterize past driving segments); 2) how to embed the recognized results into PEMSs to seek better performance enhancements; 3) how to select the length of moving horizon for data collection and the duration of output recognized patterns to avoid frequently driving patterns switches. Once the recognized driving patterns are obtained, target EMS was selected from several pre-optimized EMSs to be effective in distributing power flows until the next time instant for updating recognized driving patterns.

\subsubsection{Other prediction objectives}

Except for prediction objectives discussed above, upcoming traffic signal information (e.g. traffic light distributions) have shown their great potential in reducing fuel consumptions [33], [34] for PHEVs. Consequently, authors in [35] proposed a signal-phase prediction model which used historical averaged data and real-time phase data to determine the probability of upcoming traffic lights. The predicted information was embedded into a best velocity planning framework to avoid unnecessary stops and idling time, which turned out to give an average of 6\% improvement in FE performances.

Alternatively, as it was indicated in [36], drivers' driving styles, which were interpreted as the habits where drivers operated vehicles according to various driving scenarios, play an important role in vehicle energy management. For example, compared to eco-drivers, there were larger chances for aggressive drivers to manipulate vehicles in a more casual manner, such as over-speeding, abruptly lane changing and braking pedals abusing [37]. Consequently, such driving style will greatly increase the driving risks (e.g. traffic accidents) as well as fuel consumptions. It was found that aggressive driving styles contributed to $68 \%$ and $47 \%$ more fuel consumptions in the conventional ICE-based vehicles in urban and rural roads, respectively [38]. Similarly, previewed knowledge of driving styles can improve the performance of EMSs for HEVs/PHEVs [1]. Driving style information was embedded into an EMS for hybrid truck [39] and the recognized results of driving styles are used to explore the better performance of estimation of remaining range for PHEVs [40]. However, the precise relationships between FE and driving styles still need to be further investigated under various driving scenarios [36]. Moreover, the proper way of combination with preview knowledge on recognition results and corresponding PEMSs is still a challenging issue to be settled. Several driving prediction objectives and corresponding literatures discussed in Section 2.1 are summarized in table I. In the following parts of section 2, several specific future driving conditions (FDCs) prediction approaches are reviewed in detail including their mathematical principles, typical applications and relative comparisons. 
TABLE I

DRIVING PREDICTION OBJECTIVES AND CORRESPONDING REFERENCES

\begin{tabular}{ccc}
\hline \hline Objectives & Ref. & Sum \\
\hline Velocity & $\begin{array}{c}{[11],[13],[19]-[21],[23],} \\
{[33]-[35],[41]-[66]}\end{array}$ & 35 \\
\hline Acceleration & {$[19]-[21],[23],[46],[47],[49]$,} & 9 \\
\hline Power demand & {$[26],[27],[51],[68]-[73]$} & 9 \\
\hline SoC reference & {$[12],[13],[20],[29],[34],[68]$,} & 12 \\
\hline Driving Pattern & {$[30]-[32],[39],[40],[80]-[85]$} & 11 \\
\hline Drivers' driving styles & {$[36]-[38],[40]$} & 4 \\
\hline Traffic flow speed & {$[13]$} & 1 \\
\hline Traffic signal phase & {$[35]$} & 1 \\
\hline Traffic congestion \\
level & {$[86]$} & 1 \\
\hline Future road grade & {$[59],[87]$} & 2 \\
\hline Distance until charge & {$[28],[88],[89]$} & 3 \\
\hline $\begin{array}{c}\text { Movements of } \\
\text { preceding vehicles }\end{array}$ & {$[90]$} & 1 \\
\hline \hline
\end{tabular}

\subsection{Artificial Intelligence based methods}

AI based methods such as Artificial Neural Networks (ANNs), Machine learning (ML), decision tree algorithm (DTA) and support vector machine (SVM), have strong ability in establishing complicated relationships between inputs and outputs by the "learning" process. Moreover, they can be further categorized into two sub-parts. The first sub-part is called "recognition-based" prediction and the diagram of main procedure of this approach is shown in Fig. 2.

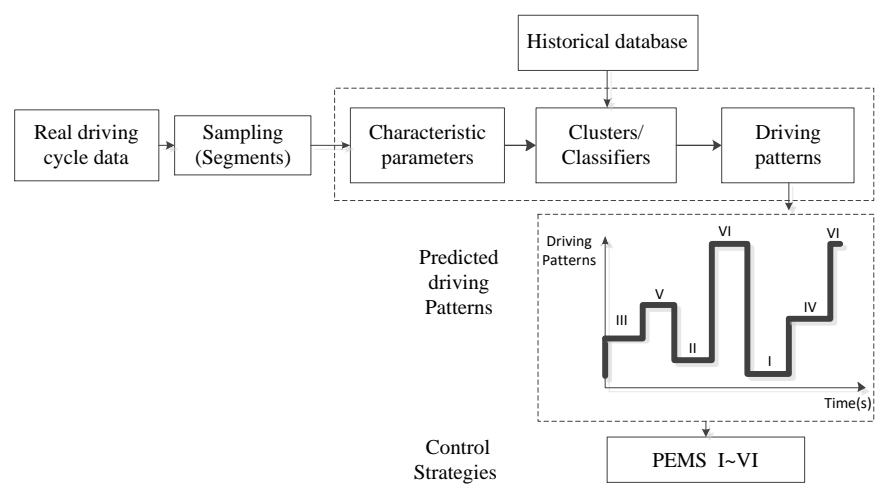

Fig. 2. Block diagram of recognition based prediction
Firstly, various clustering methods (e.g. k-means approach etc.) are used to partition historical data into several groups, where driving cycle segments with similar characteristic parameters (e.g. average acceleration, max velocity and average positive power demand etc.) are classified into same groups. Each group represented one type of pre-designed driving patterns (e.g. urban congested, urban normal, suburban, highway..., denoted as I VI, etc.). Secondly, real driving data under sufficient duration (e.g. 100-150s), which contained information on recent driving conditions, were sampled and stored to calculate corresponding driving characteristic parameters. These calculated parameters were imported into well-trained classifiers (e.g. SVM, NN etc.) to obtain driving patterns results on current segment. Finally, corresponding control strategies (e.g. PEMS I VI) designed for different driving patterns are executed according to the recognition results.

In recent years, many attempts have been made using recognition based prediction. The main research focus on this approach is the tradeoff between recognition accuracy and computation burden. The reduced number of characteristic parameters for classification can shorten the computation time making the algorithms more suitable for online applications. Among these researches, 62 [83], 40 [82], 17 [81] and 14 [86] characteristic parameters are used to characterize a specific driving cycle. This number is reduced to only 3 or 2 in [91] and [39] respectively. Especially in [66], six original characteristic parameters composed of three new parameters through linear mapping approach and the new composed parameters contained more than $90 \%$ information of variance in original data. 
Therefore, based on composed parameters, a hierarchical cluster is built to extract the characteristic parameters from real driving cycles and then SVM is used to generate the driving patterns.

The second sub-part of AI-based approach is called "experience-based" prediction whose procedure is shown in Fig. 3. In order to simplify the illustration process, a NN-based predictor, which is composed of input layer, hidden layer and output layer, is used here as a representative of AI-based prediction models. In Fig. 3, $H_{m}, H_{p}, t_{k}$ denote input horizon, output (prediction) horizon of prediction models and current time instant, respectively. $X_{i}$ and $Y_{j}$ denote input and output (predicted) variables, respectively, where $i=1,2, \ldots, \mathrm{m} . \mathrm{j}=1,2, \ldots, \mathrm{p}$.

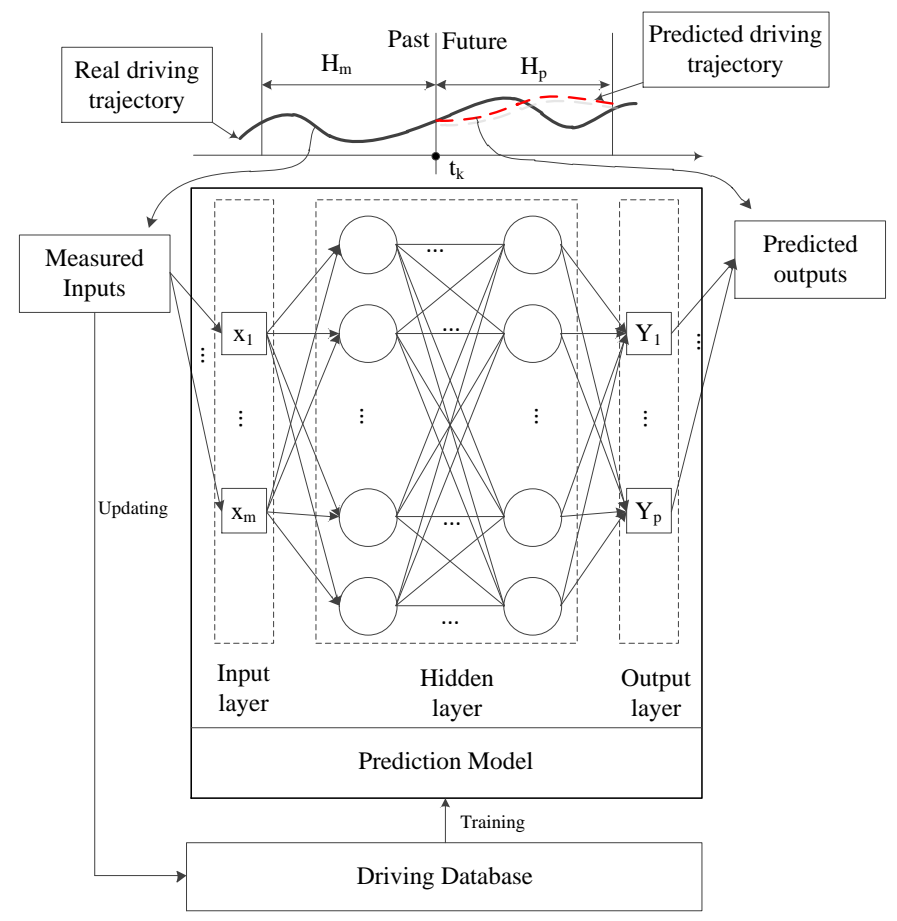

Fig. 3. Block diagram of experience based prediction

In this approach, prediction models (also known as predictors) were defined as one or more specific time-series forecasting functions. By importing current and historical data as input variables into these models, output variables (in time sequences) could be forecasted within prediction horizons. Due to uncertainty and complexity of realistic driving scenarios, future driving conditions were hard to describe accurately by traditional time-series forecasting models, which had relatively fixed structures, such as linear regression models (LRM) or autoregressive integrated moving average (ARIMA) models. For that reason, AI-based data-driven prediction models were widely adopted by researchers, which had flexible structures and could obtain necessary characteristics from training dataset automatically as long as specific training rules (e.g. activation functions, maximum training errors etc.) were established. However, it should be noted that there always existed differences between training database and real driving data. Therefore, corresponding prediction errors would dramatically increase when such discrepancy have reached certain level. In that case, updating mechanism should be established to introduce novel driving characteristics to prediction models by adding newly-measured data into training database and then retraining prediction models so that corresponding errors could be compensated.

Establishing prediction model is the most challenging task of "experience-based" approaches. Once the structure of prediction models (e.g. number of input and output variables etc.) are determined, corresponding parameters (e.g. threshold and weights etc.) are necessary to be trained by large amount of samples (from historical database) until the requirements of minimum training errors are satisfied. In the practical applications, prediction models often described complex, multivariable, non-linear relationships and therefore, such timeconsuming training processes are often finished offline. 
Some researchers have already built prediction models with this approach. For example, in [92] back-propagation neural network (BPNN) is used to establish the trip condition prediction model to obtain future velocity profile considering both traffic factors and non-traffic factors. In [93], Non-linear Autoregressive Neural Network (NARNN) combined with the moving window method is proposed to forecast future speedtime series, by updating the training database with newlymeasured data automatically. RBF-NN is used to predict the future distribution of torque [44]. From the experimental results, the proposed algorithm can achieve an average value of $89.7 \%$ fuel economy of DP-based algorithm (upper limit), which are higher than the figure of rule-based algorithms, $76.2 \%$.

Two types of AI-based prediction approaches, "recognitionbased" and "experience-based" prediction, shared the same operation framework ---“offline training” and "online predicting". However, there are two major differences between them. In "recognition-based" prediction approaches, the number of input variables is larger than that of "experience-base" prediction approaches (e.g. 100-150 vs. less than 10, respectively). The reason is, in the former approaches, it is necessary to store larger amount of data to ensure the completeness and recognition accuracy of current driving segment, while there is no need to calculate characteristic parameters in the latter approaches. Besides, the outputs of "recognition-based" prediction model are driving patterns which are used as switches to select proper pre-designed PEMSs according to the recognition results. However, the outputs of the "experience-based" prediction model are physical quantities like velocities, accelerations or power demands and these predicted profiles were regarded as the measured disturbances, which were imported into online optimizers to find satisfied solutions within prediction horizons.

\subsection{Markov based methods}

Markov chain is used to describe stochastic sequences of possible events in which the probability of each event depends only on the state obtained in the previous event. Taking a discrete finite state-space Markov chain as an example, there is a stochastic process $\left\{\chi_{n}: \mathrm{n} \in \mathrm{N}\right\}$, where all the possible values of $\chi_{i}$ formed a countable set $S=\left\{\mathrm{s}_{0}, \mathrm{~s}_{1}, \ldots, \mathrm{s}_{m}\right\}, \mathrm{m} \in \mathrm{N}$, called state space of this chain. Therefore, for this stochastic process $\left\{\chi_{n}\right\}$ and state space $S$, the next state depends on the current and the past $r-1$ states (Markov property):

$$
\begin{aligned}
& \operatorname{Pr}\left(\chi_{n+1}=s_{n+1} \mid \chi_{n}=s_{n}, \chi_{n-1}=s_{n-1}, \ldots, \chi_{0}=s_{0}\right)= \\
& \operatorname{Pr}\left(\chi_{n+1}=s_{n+1} \mid \chi_{n}=s_{n}, \chi_{n-1}=s_{n-1}, \ldots, \chi_{n-(r-1)}=s_{n-(r-1)}\right)
\end{aligned}
$$

where $r$ is the order of this Markov chain. Specifically, when $r$ equals to 1, such Markov chain is called "one-order" Markov chain, where the next state depends only on current state.

$$
\begin{aligned}
& \operatorname{Pr}\left(\chi_{n+1}=s_{n+1} \mid \chi_{n}=s_{n}, \chi_{n-1}=s_{n-1}, \ldots, \chi_{0}=s_{0}\right)= \\
& \operatorname{Pr}\left(\chi_{n+1}=s_{n+1} \mid \chi_{n}=s_{n}\right)
\end{aligned}
$$

In this way, probability of transition from state $i$ to state $j$ after one step can be marked as:

$$
p_{i j}=\operatorname{Pr}\left(\chi_{n+1}=s_{j} \mid \chi_{n}=s_{i}\right) \quad i, j \in m
$$

where $p_{i j}$ is the element lying in the $i$ th row and $j$ th column of Transition Probability Matrix (TPM), marked as $P$.

Once TPM is established, given state $s_{i} \in S$, corresponding probability vector $v(k)=\left(p_{i 1}, p_{i 2}, \ldots, p_{i m}\right)$ can be obtained, which defined probability distributions of all possible 
transitions starting from state $s_{i}$ at time step $t=k$ to state $s_{r}, r \in 1,2, \ldots, \mathrm{m}$ at time $\operatorname{step}_{t}=k+1$. Therefore, one step-ahead probability vector $v(k+1)$ can be written as:

$$
v(k+1)=v(k) \cdot P
$$

Similarly, for $H_{p}$ steps ahead, the probability vector $v\left(k+H_{p}\right)$ is denoted as

$$
v\left(k+H_{p}\right)=v(k) \cdot P^{H_{p}} .
$$

The overall procedure of making prediction by Markov chain is shown in Fig. 4. There are four main steps in the prediction process. Step I: establishing TPM by statistical approaches; Step II: encoding inputs variables (e.g. velocity, acceleration, power request etc.) into states; Step III: generating the predicted states; Step IV: decoding predicted states into real physical values. Detailed information of general procedure of Markov chain prediction can be found in [45].



Fig. 4. Block diagram of Markov chain based prediction

For practical applications, future probability distributions of vehicles' velocity, acceleration and drivers' power request can be predicted by Markov model. For example, in [38], [69], [94] where a Markov chain was used to establish the future distribution of velocity and acceleration in order to predict the future velocity $((\mathrm{V}, \mathrm{A})-\mathrm{V}$ distribution). In addition, future vehicle power demand sequence was generated by Markov chain automatically, where input variables are current power demand [27], [69] (P-P distribution) or current power demand and velocity [51], [70] ( (P,V)-P distribution).

Besides, in [95], a Hidden Markov model (HMM) was proposed to generate future vehicles velocity and position sequence based on current engine torque and velocity. The results showed that proposed tube-based MPC PEMS could improve fuel economy by $0.65 \%$ than the deterministic MPCbased PEMSs and maintain SoC within the pre-designed boundaries. Moreover, Markov chain was used to model the drivers' behaviors to give the power request from driver at next state [26]. Corresponding results showed that compared with the fuel consumed by classical MPC-based EMSs, the best possible FEI on two different real world driving cycles achieved by MPC controller with full previewed knowledge were $8.19 \%$ and $12.73 \%$, respectively, while the results of the proposed stochastic MPC controller with learning algorithm were $8.04 \%$ and $11.34 \%$. This means the proposed PEMS could achieve a significant FEI than classic MPC controller, while its result is very close to the best possible benchmark. From these researches, it can be found that accuracy of Markov based prediction algorithms and the performances of corresponding PEMSs can be guaranteed. However, if real driving conditions changed dramatically or prediction model did not hold Markov property, corresponding improvements of may be lost [52].

\subsection{Exponentially decreasing model based methods}

According to frequent variations of torque demand in a real 
driving cycle, assumption of a decaying torque demand was more reasonable than a constant torque assumption, which was testified by H. Ali Borhan et al.in their researches [96].

Exponentially decreasing model (EDM) was based on the assumption that drivers' torque demand in the future will drop exponentially within prediction horizon. Therefore, this model can be described by (8). Combining (8) with the discrete form of vehicle dynamics model (9) and by numerical integration, the predicted value of vehicle velocity can be given in (10).

$$
\tau_{\text {drive }}[(k+i) T]=\tau_{\text {drive }}[k T] e^{\left(\frac{-i T}{T_{d}}\right)} \quad i=1,2, \ldots, H_{p}
$$

$$
\begin{aligned}
& m_{v} \frac{v[(k+i) T]-v[k T]}{i T}=F_{t}[(k+i) T]-\left(F_{a}(k T)+F_{r}(k T)+F_{g}(k T)\right) \\
& F_{t}(k T)=\frac{\tau_{\text {drive }}}{r_{w}}(k T)
\end{aligned}
$$

$$
v[(k+i) T]=v[k T]+\frac{\sum_{i=1}^{p}\left[\frac{1}{r_{w}} \cdot \tau_{d r v i v}(k T) e^{\left(-\frac{i T}{T_{d}}\right)}-F_{a}(k T)-F_{r}(k T)-F_{g}(k T)\right] \cdot i T}{m_{v}}
$$

where $\tau_{\text {drive }}(k T)$ is torque requests from driver at the

beginning of the prediction horizon, $T$ is sampling time, $T_{d}$ is decay-determined parameter, $H_{p}$ denotes the prediction horizon, and $r_{w}$ denotes the wheel radius.

Here, a smaller $T_{d}$ (faster decay) is selected for describing larger torque demand. The physical reason behind that is the periods of large torque demand is very short in typical driving cycles. In other words, statistically, large torque demand is not expected to last long.

In [53], this model was used to predict future velocity of a PHEV, and prediction results were utilized to develop a MPC based PEMS with a hybrid energy storage system. In [54], [55], this model was used to provide the prediction of future vehicle velocity and was implemented in the Nonlinear MPC based
PEMS framework. In [23], three types of velocity predictors established by RBF-NNs, one, five order Markov chain and EDM were embedded into the MPC based PEMSs framework. From the results of the comparative studies, the prediction error of RBF-NNs predictor was $32.7 \%, 38.0 \%$ and $26.9 \%$ smaller than that of EDM, one and five order Markov based approaches, whose fuel consumption was $9.7 \%, 16.3 \%$ and $6.9 \%$ lower than that of other predictors respectively. Moreover, the calculation time of RBF-NN predictor based PEMS was $0.208 \mathrm{~s}$, which was much shorter than one and five order Markov chain, 1.647s and 2.979s respectively, and longer than that of EDM predictor, 0.032s. Consequently, it can be concluded that RBF NN-based velocity predictors can offer the best overall performance within acceptable calculation time.

\subsection{Telematics technique based methods}

Thanks to the development of telematics techniques (GPS and navigation system etc.), predictions can be made more accurately with previewed trip knowledge. From previous researches, it can be seen that PEMSs outperforms than many Non-Predictive Energy Management Strategies (N-PEMSs). However, such advantages can be compromised or even lost if EMSs were proposed based on the inaccurate prediction results [34], which were mainly caused by uncertain traffic factors (e.g. traffic light signal distribution and traffic congestion level etc.) and road-related information (e.g. future terrain and speed limits etc.).

For example, in [75], authors presented a PEMS for HEVs with preview of road grade. Double-layer PEMSs were established in [61], [74] and [78] using the future driving information like traffic lights positions, speed limits and traffic 
congestion level. Trip-preview based information was used to construct the driving cycle [10], [57] and identify the route features [79] from historical and real-time data.

An online intelligent energy management controller was built by dual-NNs, which was associated with precise or estimated the preview knowledge of trip length and duration [71], [97]. And the simulation results showed that with precise previewed knowledge, the proposed online controller can achieve $3.96 \%$ and $2.79 \%$ of fuel economy improvements (FEI) on 4 LA92 and 3 REP05 driving cycles, respectively. While $4.7 \%$ and $2.8 \%$ of FEI were achieved with estimated preview knowledge on 4 Artemis driving cycle and 7 NEDC (New European Driving Cycle) driving cycles, respectively. In [87], [98], C. Zhang.et al. studied potential impacts of terrain previewed knowledge bringing to the corresponding performance among ECMS and DP based EMSs. The simulation results showed that on average, hilly terrain previewed knowledge could bring 1\%-4\% more FEI than those without previewed knowledge. Moreover, terrain preview can decrease the energy flows to and from battery packs, which increased the battery's life.

An optimal velocity planning algorithms is scheduled based on the preview of traffic signal distribution to reduce the fuel consumption in [35], where the proposed approach can reduce fuel consumption by $6 \%$ with the combination of real-time information. Similarly, in [33], a combination of upcoming traffic signal information and vehicle's adaptive cruise control system were proposed to reduce the idle time of the engine and improve the overall FEI. In simulation, the fuel consumption was brought down by $47 \%$ through a sequence of 9 traffic lights.
However, such giant FEI may be achieved in future for practical application when the advanced intelligent traffic intersection control systems were available. Markov chain based road grade predictor [59] was built for proposing PEMSs with route based information (e.g. vehicle location, traveling direction, and terrain information etc.). Corresponding results showed that the proposed strategies consumed $4.3 \%$ more fuel than the best benchmark (DP-based EMS) but $8.2 \%$ less fuel than ECMSbased EMS.

\subsection{Other methods}

Apart from the above-discussed prediction approaches, other prediction methods were also explored by researchers. For example, a fast dual-loop Nonlinear PEMSs for HEVs was proposed [99], where the inner loop aimed at tracking the reference trajectories based on the knowledge of predicted driving cycle. An ARIMA based method was proposed to forecast the future road grade [100]. The root mean square error (RMSE) of 10-second road grade forecast is as low as 0.01 degree and corresponding fuel economy was improved by 4.7\%. In [63], a gain scheduled driver model (GSDM) was used for predicting the future driving profile. From the results of simulations, the proposed EMS could obtain about $84 \%$ of best possible FEI results due to the use of prediction results. In [101], authors demonstrated a method to identify commuter routes from historical driving data using hierarchical agglomerative clustering method (HACM). In [16], a prediction method was proposed concentrating on the frequency distribution of vehicles future position. Moreover, several DPR and classification methods were proposed based on analytical LVQ-NN [102], similarity degree (SD)[103], fuzzy logic (FL) 
[31], [62], Probabilistic support vector machine (PSVM) [104] and Kalman filter [105].

Based on the comprehensive review of specific FDCs

prediction methods in Section 2, a classification of these methods is proposed and corresponding references are summarized in Table II.

TABLE II

SUMMARY OF SPECIFIC APPROACHES FOR FDCS PREDICTION

\begin{tabular}{|c|c|c|c|}
\hline Classification & Sub-classification & Ref. & Sum. \\
\hline \multirow[b]{2}{*}{ AI approaches } & Recognition based & [39], [66], [81]-[86], [91] & 9 \\
\hline & Experience based & $\begin{array}{c}{[18],[41]-[44],[68],[69],[80],[92],[93],} \\
{[106]-[109]}\end{array}$ & 14 \\
\hline \multirow{6}{*}{ Markov approaches } & $(\mathrm{V}, \mathrm{A})-\mathrm{V}$ distribution ${ }^{1}$ & [19]-[21], [45]-[50], [62], [67] & 11 \\
\hline & $\mathrm{P}-\mathrm{P}$ or $(\mathrm{P}, \mathrm{V})-\mathrm{P}$ distribution ${ }^{2}$ & $\begin{array}{c}{[26],[27],[51],[64],[70],[73],[93],} \\
{[110]}\end{array}$ & 8 \\
\hline & (V-Road Grade) 2D distribution & [52] & 1 \\
\hline & HMM Driver Torque distribution & {$[95]$} & 1 \\
\hline & Diving behaviors distribution & [111] & 1 \\
\hline & GPS coordinates distribution & [89] & 1 \\
\hline \multirow{2}{*}{$\begin{array}{l}\text { Telematics } \\
\text { approaches }\end{array}$} & Uncertain traffic factors & [34], [35], [58], [60], [61] & 5 \\
\hline & Road-related information & $\begin{array}{c}\text { [10], [11], [34], [56], [57], [71], [74], [75], } \\
\text { [78], [79], [87], [97], [98], [112], [113] }\end{array}$ & 15 \\
\hline \multirow{10}{*}{ Other approaches } & Frequency Distribution Analysis & [16] & 1 \\
\hline & ARIMA model & [100] & 1 \\
\hline & Extended Kalman Filter & {$[105]$} & 1 \\
\hline & SVM classifier & [104] & 1 \\
\hline & Similarity degree classifier & [103] & 1 \\
\hline & $\begin{array}{l}\text { Real world driving cycle construction based on statistical } \\
\text { approaches }\end{array}$ & [102] & 1 \\
\hline & Fuzzy Logic based driving pattern recognizer & [31] & 1 \\
\hline & Hierarchical agglomerative clustering route identification & [101] & 1 \\
\hline & Gain scheduled driver model & [63] & 1 \\
\hline & High power demands identification & [114] & 1 \\
\hline EDM approaches & - & {$[23],[53]-[55],[96]$} & 5 \\
\hline
\end{tabular}

${ }^{1,2} \mathrm{~V}, \mathrm{~A}, \mathrm{P}$ refers to vehicle velocity, acceleration and power demand from external environments or drivers' requests.

\section{FURTHER DISCUSSION}

In this section, issues like comparative study and suitable application scenarios of each prediction method, factors causing mis-predictions, the mechanism how these factors affect the quality of predictions and methods to cope with mispredictions are discussed in detail.

\subsection{Comparative study of FDCs prediction methods}

A comparative study of driving prediction methods will be conducted in this subsection through their mathematical principles, application complexity, (e.g. data demands and computation burdens), prediction accuracy and robustness.

\subsubsection{Mathematical principles}

The mathematical principle of AI-based prediction approaches is to find the nonlinear, multi-variate and constrained functions to describe the relationships between measured inputs and predicted outputs. Such functions can be obtained by adjusting weights to minimize the error between 
the actual and predicted output patterns of a bunch of training data (training process). The well-trained prediction model are used for generating the future sequences of driving conditions.

Markov based prediction approaches are used for modeling stochastic processes which satisfy the Markov property ("memoryless" property). Once TPMs established by statistical approaches and input states are determined, one can generate the probability distribution of next states. Finally, the deterministic prediction outputs can be decoded from the probability distribution of next states by Monte Carlo simulation, mathematical expectation or probability maximization approaches [52].

EDM based prediction approaches are based on the simple physical model (8) that the torque requests from drivers are decreasing exponentially with time. The only parameter needs to be tuned before application is decay-determined factor $T_{d}$.

Telematics technique based approaches take advantage of various types of real-time route information (road slopes, speed limits, travel distance etc.) from GPS, Intelligent Transportation Systems (ITS) and make predictions by integrating this information into well-trained time series forecasting models (e.g. LRM, ARIMA, and Support Vector Regressions (SVR) etc.).

\subsubsection{Application complexity}

TPM is the core part in Markov prediction model. For practical applications, whether standard driving cycles, like FTP-75 cycle (for Federal Test Procedure), NEDC and UDDS, or real world experimental data can be used to establish TPMs. In order to ensure the accuracy of prediction results, the database used for establishing TPMs should be abundant to cover as much driving conditions as possible, which causes large data demand and computation burden. Besides, the input variables compose of the basic elements in Markov chain--states. Moreover, the increasing number of states and orders of Markov chains will increase the model resolution, resulting in high prediction accuracy. However, the higher complexity and large amount of data demand brought by them will also increase the computation burdens for real time applications.

The procedures of implementing AI-based prediction approaches can be divided into three parts, determining the structure of prediction models, offline training and online predicting. Taking NN-based approach as an example, the number of input and output variables, the structure and number of neurons and layers, have to be pre-determined according to the complexity of concerned problems. Then, during the offline training processes, large amount of samples are used to train to prediction functions, which can be very time-consuming. Besides, if the amount of training data is too small or the structures of prediction models are designed too complex, the "overfitting" problem would cause many noises in the online prediction process and thus decrease the prediction accuracy for practical applications. Moreover, if the training error is set too small, it may take long time for the training process to be converged.

Unlike AI-based approaches, prediction models of EDM based approaches have the fixed structures. Once the only parameter $T_{d}$ is well-tuned within available dataset, this timesaving model can be easily used for online applications and its data requirement is smaller than other prediction models.

Similar as EDM based approaches, generally, telematics 
technique based approaches are established based on various time series forecasting models which have their own structures. The only work needed to be done before application is to choose proper parameters (e.g. the order of ARIMA model etc.) regarding the complexity of studied problems. Statistical approaches, like principal component analysis and correlation analysis, may be used for finding the relationships between various type of traffic information and predicted values.

\subsubsection{Prediction accuracy and robustness}

Compared with other types of prediction approaches, prediction accuracy and robustness of EDM based approaches are limited due to the discrepancy between its fixed prediction model and rapidly changeable external driving conditions.

If the studied processes do not fit in with Markov property, the prediction accuracy of Markov based approaches will be greatly influenced. Besides, if uncertain disturbances (e.g. start/stop, traffic accidents etc.) or new driving conditions happen during real applications, the prediction performance will dramatically drop because forecasted behaviors may not comply with historical laws under such circumstances.

Generally, AI based approaches can handle more input variables (e.g. more than three) than Markov based approaches (e.g. no more than two), which means more useful information is used to help improving the performance of predictions. For practical applications, total database are usually divided into training parts (e.g. 70\%-80\% of total database) and testing parts (e.g. 20\%-30\% of total database), where the former is used for training prediction models while the latter for testing their correctness. However, in this situation, the prediction models perform well only when encountering the similar driving situation as training database but when it comes to changeable real driving conditions, corresponding good performances may be lost.

Telematics technique based approaches have the potential to provide the most accurate prediction results, because real time traffic situation are considered when making predictions. However, advanced traffic control systems are at the early age of development. Besides, associated with handling large amount of data, the exponentially increasing computational burdens propose higher requirements for high-performance onboard processors. These are two major factors that will influence the performances of telematics technique based approaches.

\subsection{Application scenarios of each prediction method}

Predictions based on EDM models are time-saving because its prediction model is fixed and simple. However, such model remains unchanged when external driving conditions dramatically change, which leads to unreliable results for real driving conditions. Consequently, it is suitable to be a good benchmark in simulations or stable driving environments where driving conditions do not change rapidly.

For private vehicles, Markov based prediction methods are not suitable for applying in urban area where most vehicles are often in the "stop-and-go" mode, traffic signals are changing with time, vehicles' velocity are highly affected by the movements of preceding vehicles, leading to the difficulties in accurately predicting vehicle's velocity. Predictions based on historical data are more reasonable for public service vehicles, like city buses [19]-[21], [46], [48], [66] and trucks [39], [63] etc., because their working conditions and commuter routes are 
relatively fixed and repeated. Therefore, historical data can reflect their common behaviors with high reliability. Additionally, Markov based approaches are also suitable for highway driving conditions. It should be noted that 1) external driving environments in highway do not change as rapidly as in urban areas, 2) the movements of vehicles are relatively similar on highway than in urban areas, for example, most vehicles are cruising around the upper speed limits in majority of driving time, their velocity will be reduced at every entrance and exit and they will stop at every toll gate, which means, statistically, vehicles in highway driving conditions will follow the similar driving patterns as recorded in historical database. In that case, TPMs established by such database can effectively reflect future probability distribution of vehicle speed, resulting in higher prediction accuracy.

The potential of integrating accurate traffic information (e.g. traffic speed, road slope and traffic lights distribution etc.) to reduce the future uncertainties makes AI based methods perform better than Markov based methods in urban areas. However, the time-consuming model training processes should be finished offline. In order to make the model adapt to the changeable external driving conditions, online learning/training algorithms should be introduced, which will additionally increase computation burdens. For vehicles having relatively fixed operation routes, this approach may have good performance in real time because the offline well-trained models can perform well in most of their driving conditions. Similar to Markov based approaches, AI based approaches are suitable for applications in highway conditions.
If vehicles are assumed to be operated in an urban area with heavy traffic, short-term road grade preview or even the current road grade information would be of great help for designing PEMSs, since vehicles' average velocity is low but have many transients, which is hard to be accurately predicted by AI-based or Markov based approaches. Moreover, advanced wireless communication systems like Vehicle-to-Vehicle (V2V) and Vehicle-to-Infrastructure (V2I) communication also provide the data exchanging and sharing platforms for vehicles on the specific roads. The shared information between vehicles and infrastructures are useful to gain the information of potential movements of surrounding vehicles, whose results can be utilized for the velocity prediction during traffic congestion periods. Besides, in suburban and highway driving conditions, this approach may not be suitable for prediction because corresponding advanced intelligent traffic devices may not be well-developed in these areas.

According to discussions in section 3.1 and 3.2, benefits, drawbacks and suitable application scenarios of several types of prediction approaches are summarized in Table III.

\subsection{Approaches to cope with mis-prediction}

Apart from selecting proper prediction horizon, there are many other approaches that can be found in existing literatures to cope with mis-predictions. In this sub-section, several accuracy-affecting factors are firstly analyzed and then different types of mis-predictions caused by these factors and their influences on corresponding PEMSs are indicated. Finally, approaches for improving prediction accuracy and robustness are discussed. 
BENEFITS, DRAWBACKS AND SUITABLE APPLICATION SCENARIOS OF PREDICTION APPROACHES

\begin{tabular}{|c|c|c|c|}
\hline Method & Benefits & Drawbacks & Suitable application scenarios \\
\hline EDM & $\begin{array}{l}\text { 1. Easy and robust to implement; } \\
\text { 2. Less computation burden and data } \\
\text { requirements. } \\
\text { 3. Good benchmark. }\end{array}$ & $\begin{array}{l}\text { 1. Unchanged model structures do not } \\
\text { have good adaptation to various external } \\
\text { environments; }\end{array}$ & $\begin{array}{l}\text { 1. Stable real driving conditions (highway) or } \\
\text { simulation environments. }\end{array}$ \\
\hline AI & $\begin{array}{l}\text { 1. Gaining non-linear multiple-variable } \\
\text { relationships ( more than } 3 \text { inputs); } \\
\text { 2. Potential of integrating preview } \\
\text { knowledge; } \\
\text { 3. Strong learning capacity. }\end{array}$ & $\begin{array}{l}\text { 1. Training processes are time- } \\
\text { consuming. } \\
\text { 2. Model complexity, "over-fitting" and } \\
\text { "convergence" problem; } \\
\text { 3. Training database could not cover } \\
\text { every possible driving conditions. }\end{array}$ & $\begin{array}{l}\text { 1. In urban areas, AI based approaches } \\
\text { associated with online learning algorithms can } \\
\text { provide more accurate results for private } \\
\text { vehicles. } \\
\text { 2. For commuter and public service vehicles in } \\
\text { urban/suburban/highway areas. }\end{array}$ \\
\hline Telematics & $\begin{array}{l}\text { 1. More accurate real-time driving data } \\
\text { available; } \\
\text { 2. Potential of prediction in rush hour and } \\
\text { traffic congestion conditions. } \\
\text { 3. Potential of applying EMSs on whole } \\
\text { traffic flows. }\end{array}$ & $\begin{array}{l}\text { 1. No common method for integrating } \\
\text { telematics data into the PEMS; } \\
\text { 2. Large computation burden; } \\
\text { 3. Early stage of ITS and traffic flow } \\
\text { modeling techniques; }\end{array}$ & $\begin{array}{l}\text { 1. Urban areas, especially for rush hours and } \\
\text { traffic congestions on condition that } \\
\text { corresponding information are available. }\end{array}$ \\
\hline Markov & $\begin{array}{l}\text { 1. Less dependency on preview knowledge; } \\
\text { 2. Suitable for modeling stochastic process. } \\
\text { 3. Reasonable accuracy on similar driving } \\
\text { conditions. }\end{array}$ & $\begin{array}{l}\text { 1.Lower prediction accuracy for rapidly } \\
\text { changeable driving conditions; } \\
\text { 2. Difficulties in integrating with real- } \\
\text { time traffic information. } \\
\text { 3. Poor performance for processes do } \\
\text { not comply with Markov property. }\end{array}$ & $\begin{array}{l}\text { 1. Commuter or public service vehicles in } \\
\text { urban/suburban areas, where their working } \\
\text { conditions are repetitive and comply with } \\
\text { historical measured data. } \\
\text { 2. Highway area. }\end{array}$ \\
\hline ARIMA & $\begin{array}{l}\text { 1.Advances in forecasting time-series } \\
\text { sequences; } \\
\text { 2. Potential to reduce "non-stationary" } \\
\text { characteristics in data; }\end{array}$ & $\begin{array}{l}\text { 1. Less adaptability due to relatively } \\
\text { fixed structures; } \\
\text { 2. Huge dependency on historical } \\
\text { database due to its data-driven property. }\end{array}$ & $\begin{array}{l}\text { 1. Suitable for rural, mountainous area, where } \\
\text { modern telematics technique is at early stage } \\
{[100] \text {. }}\end{array}$ \\
\hline GSDM & $\begin{array}{l}\text { 1.Release high computation and storage } \\
\text { burden to be easily used online; } \\
\text { 2. Adaptability to real driving conditions } \\
\text { were ensured by online RLS approaches. }\end{array}$ & $\begin{array}{l}\text { 1. Only a single value of vehicle } \\
\text { velocity at every stopping position was } \\
\text { stored and corresponding prediction } \\
\text { accuracy might be compromised. }\end{array}$ & $\begin{array}{l}\text { 1. Suitable for vehicles having repeated } \\
\text { operations on the same roads (e.g. buses, } \\
\text { garbage trucks etc.)[63]. }\end{array}$ \\
\hline HACM & $\begin{array}{l}\text { 1. Unsupervised learning technique, where } \\
\text { the number of clusters need not to be } \\
\text { specified in advance; } \\
\text { 2. Both driving conditions and } \\
\text { uncertainties of the exact final position } \\
\text { could be modeled. }\end{array}$ & $\begin{array}{l}\text { 1. Highly dependent on servers where } \\
\text { complicated computation and large data } \\
\text { storage space were required. } \\
\text { 2. Highly dependent on corresponding } \\
\text { route-based techniques, like route } \\
\text { identification approaches. }\end{array}$ & $\begin{array}{l}\text { 1. Suitable for commuter routes, where } \\
\text { predictions based on historical data are reliable } \\
\text { [101]. }\end{array}$ \\
\hline $\begin{array}{l}\text { Frequency- } \\
\text { based }\end{array}$ & $\begin{array}{l}\text { 1. Reduce prediction difficulty by } \\
\text { forecasting the future distribution of FDCs } \\
\text { in frequency domain rather than in time } \\
\text { domain; } \\
\text { 2. Less dependence on telematics devices. }\end{array}$ & $\begin{array}{l}\text { 1. Limitation caused by the assumption } \\
\text { that optimal costate for Hamiltonian } \\
\text { equation is constant }[16] \text {; }\end{array}$ & $\begin{array}{l}\text { 1. Proper for urban and commuter vehicles } \\
\text { especially for real-time application. }\end{array}$ \\
\hline SD & $\begin{array}{l}\text { 1. Compared to traditional DPR techniques, } \\
\text { SD is more computational efficient. } \\
\text { 2. Time-consuming training processes were } \\
\text { not required. }\end{array}$ & $\begin{array}{l}\text { 1. Weight factors of each driving } \\
\text { characteristic parameters were hard to } \\
\text { be tuned [103]. }\end{array}$ & $\begin{array}{l}\text { 1. Suitable for urban driving conditions, where } \\
\text { vehicles shared similar driving characteristics. }\end{array}$ \\
\hline FL & $\begin{array}{l}\text { 1. Less computation burden and robust to } \\
\text { external disturbances; }\end{array}$ & $\begin{array}{l}\text { 1. Parameters of membership functions } \\
\text { need to be re-tuned, when handling } \\
\text { more driving scenarios [31]. }\end{array}$ & $\begin{array}{l}\text { 1. Suitable for urban driving conditions, where } \\
\text { vehicles shared similar driving characteristics. }\end{array}$ \\
\hline PSVM & $\begin{array}{l}\text { 1. Powerful tool in recognizing unknown } \\
\text { driving patterns into several known cases. }\end{array}$ & $\begin{array}{l}\text { 1. Recognition performances highly } \\
\text { depended on several pre-set known } \\
\text { cases [104]. } \\
\text { 2. Time-consuming training process. }\end{array}$ & $\begin{array}{l}\text { 1. Suitable for realistic driving conditions where } \\
\text { large amount of training dataset is available. }\end{array}$ \\
\hline
\end{tabular}

\subsubsection{Factors causing mis-predictions}

categorized into four parts:

Factors that affect the prediction accuracy can be roughly 
It is illustrated by Hongwen $\mathrm{H}$. et al. in [49] that 1) the prediction accuracy can be affected by the length of prediction horizon and 2) prediction with variable horizon can provide $20 \%$ more accurate results than with fixed prediction horizon. In [115], a prediction horizon with non-uniform sampling times was proposed benefiting from both a short prediction horizon with small sampling instants and a long prediction horizon with large sampling instants. Corresponding MPC based PEMSs framework with proposed predictive strategy reduced the range and variation of battery power demand by $25.5 \%$ and $46.6 \%$, respectively, compared with the results from Rule-based EMSs. Generally, compared with long-term prediction, prediction with shorter horizon will be more accurate because it is less likely to encounter the external changes and its prediction results will be less affected in short period due to the inertia of systems. However, PEMSs with short-term prediction will always lead sub-optimality to the performance of proposed PEMSs. Consequently, tradeoff should be made when choosing proper length of prediction horizon.

- Prediction model distortion

Markov chain and NN based prediction models were generated by "learning" or "training" process, whose performances highly rely on the stationary database. However, real world driving conditions are complex and changing dramatically according to many uncertain factors, like the movements of pedestrians, surrounding vehicles and weather conditions etc. Therefore, such model distortion caused by the discrepancy between original database and real-time changing environments becomes another prediction accuracy-affecting factor. However, if the original database or the prediction model can be updated by online updating algorithm [51], the prediction model can "learn" new characteristics from external environments. Consequently, the adaptation for prediction models will be improved.

\section{- Prediction-assisting knowledge}

Three types of knowledge are utilized to explore their potential of accuracy improvements by researchers:

a) Knowledge from historical database. For example, in order to increase the amount of prediction-assisting information from historical database to identify Transition Probability Matrix (TPM), conventional "one-order" Markov model (2) is expanded to "high-order" Markov model (1) to increase the resolution and accuracy of prediction models. Such expansion required large amount of driving data to estimate corresponding TPMs but such sufficiently rich database cannot be guaranteed in designing processes. That is reason that more than five order Markov chain is rarely seen in corresponding researches[23], [67], [69].

b) Knowledge from traffic conditions: The influences on prediction accuracy of traffic-based preview knowledge like terrain knowledge [87], [98], traffic congestion level [61], traffic signal distribution [33], [35], [58], [61] and trip length [97],[79] were explored by researchers. Various data-fusion approaches (e.g. Dempster-Shafer (DS) and Wavelet Transform (WT) theory) are implemented to assign different weights to these accuracy-affecting factors to create multi-input prediction models.

c) Knowledge from non-traffic conditions. Non-traffic factors like weather conditions (rain, sun, wind...) [6], driving period (weekdays or weekends) [42] or even drivers' style 
(aggressive, moderate, conservative) [1] can also affect the prediction accuracy. For example, detailed expressions of aerodynamic friction $F_{a}$ and rolling friction $F_{r}$ in vehicle dynamic longitude model (1) are given in (11) and (12).

$$
F_{a}=\frac{1}{2} \rho_{a} \cdot A_{f} \cdot c_{d} \cdot v^{2}
$$

$$
F_{r}=c_{r} \cdot m_{v} \cdot g \cdot \cos (\alpha)
$$

In (9), actual air flow coefficient $c_{d}$ can be heavily affected by external unpredicted wind, the addition of cargo outside the vehicle and driving with windows down [116]. The rolling friction coefficient $c_{r}$ in (12) can increase by $20 \%$ when driving on the wet road, while this value will double when driving in sand [6], which will lead to corresponding changes to predicted values like power demand and velocity.

\section{- Suitable prediction methods}

Like analysis in section 3.2, different prediction approaches perform better in their suitable application scenarios. Besides, another factors like computation burdens and algorithm complexity should also be taken into consideration. For example, in [23], three different prediction methods were used in the same framework and the experimental results shown that prediction accuracy of RBF-NN based approach was $9.7 \%$ higher than that of EDM based approach, while its computation time $(0.208 \mathrm{~s})$ is 6.5 times of that of EDM $(0.032 \mathrm{~s})$. Under such circumstances, the former approach may be a better prediction approach in simulations where its computation time is acceptable. However, for practical applications, designers should consider the performances' limits of hardware devices and ensure prospective algorithms can completely meet the online requirements.

It is summarized in the previous part of this sub-section that there are four main factors that influenced the prediction accuracy. In the following part, the discussion will focus on what kind of influences these factors will bring to corresponding Predictive Energy Management Strategies (PEMSs).

Firstly, different types of previewed knowledge will bring different levels of impacts to corresponding PEMSs. For example, in [11], three different levels of previewed knowledge, namely level 1: the knowledge of terrain, trip length and estimated velocity, level 2: hilly terrain and distance to next charging station and level 3: without preview, are integrated into PEMSs to evaluate their different potentials of fuel saving.

The cost gaps (\%) between them and best possible benchmark are used to evaluate the performance of other control strategies. Moreover, the results showed that PEMS with level 2 of previewed knowledge saved $7.4 \%$ more fuel than PEMS with level 3 of previewed knowledge, which means the previewed knowledge of hilly terrain and distance to next charging station are significant in improving the FE. And in another case study, it is testified that estimated velocity and hilly terrain preview knowledge together will bring about up to only $1 \%$ additional fuel economy improvement (FEI). Therefore, among these prediction accuracy-affecting factors, the distance to next charging station has the most significant influence on the FE.

Secondly, impacts of various mis-predictions bringing to PEMSs are different. In order to figure out influences caused by different mis-predictions, in [116], driving-derived prediction errors (predicted velocity, traffic conditions, additional 
starts/stops, route change etc.) and vehicle parameter prediction errors (vehicle mass, drag coefficients, rolling resistance etc.), are applied to the same PEMS to explore corresponding performance changes. The results indicated that when “additional stop mis-predictions" occurs, where 1,2 or 3 unexpected stops are added into predicted driving profiles, corresponding optimal PEMS can still bring fuel consumption down by $10.8 \%, 7,9 \%$ and $5.1 \%$, respectively. Compared with 10.9\% FE improvement brought by baseline optimal PEMS, it is clear that the FE improvements are maintained under such type of mis-predictions. However, FEI under "route change" mis-predictions may be lost. For example, a $-4.3 \%$ (out of $+13.6 \%$ possible) FEI was obtained if the drive cycle is the same as expected but then is suddenly ended shortly after it has begun.

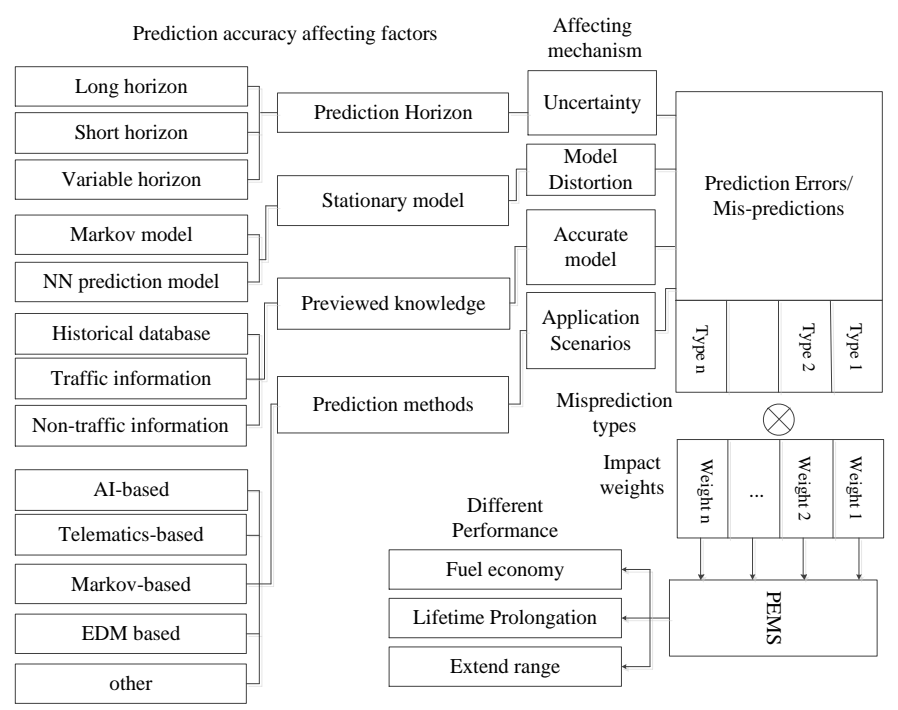

Fig.5. Summary block diagram of factors causing mis-predictions

According to these analysis, prediction accuracy affecting factors, corresponding mechanisms leading to mis-predictions and how these factors affecting the performance of PEMSs are summarized in Fig. 5.

\subsubsection{Approaches to improve prediction accuracy}

Considering different types of factors causing mispredictions, several approaches to improve the prediction accuracy were proposed by researchers.

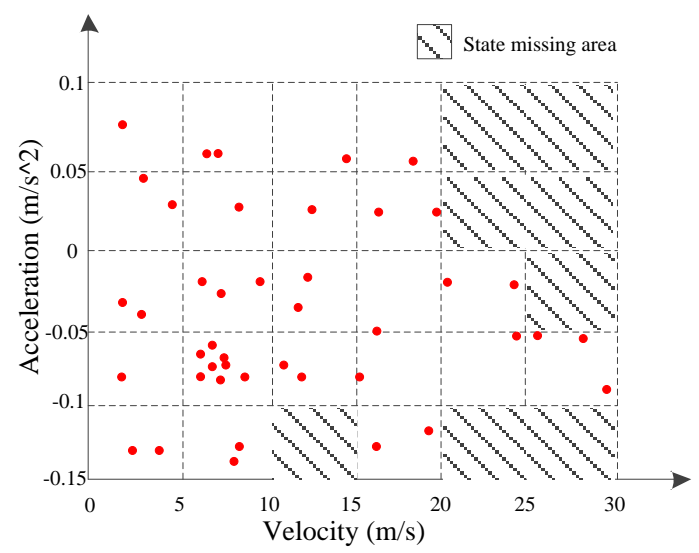

Fig. 6. State-missing phenomena in velocity-acceleration plane

Within conventional Markov based prediction model, due to the limited amount of training data, there may be some blank areas in the state plane, where no such driving conditions/states exist in training database, which can be seen in the shaded parts in Fig. 6, taking velocity-acceleration ( $\mathrm{v}-\mathrm{a})$ plane as an example. Such phenomena is called state-missing phenomena. However, in real driving world, vehicle's states will appear at any area of v-a plane. When real driving states reach in the blank areas, the prediction process will be interrupted, which directly leads to low prediction accuracy. Authors in [49] proposed an online reconstruction approach which can ensure the prediction process will be conducted continuously without state-missing. And then, analytical methods like principle component analysis and cluster analysis are implemented to form the Markov-based prediction model with variable horizon, showing $20 \%$ accuracy improving than fixed time horizon prediction.

In traditional fixed-time scale Markov based approaches 
(2) (5), future driving cycle is predicted on the basis of previous prediction results step by step, which is called "Singlescale Multi-step" (SSMS) prediction. In order to reduce the accumulation errors during such calculation processes, "Multiscale single-step" (MSSS) Markov prediction model was proposed in [50] and applied in [19], [20]. In this framework, TPMs at different time scales were built in advance and each of them reflected the probability distribution from states at $t$ to states $t+r, r=1,2, \ldots, H_{p}$, where $H_{p}$ is prediction horizon. During prediction processes, predicted value at different time scales were obtained by the value of states at $t$ and a bunch of different-time-scale TPMs. MSSS based prediction methods obtained $7.18 \%$ improvement of prediction accuracy than SSMS [50].

Some approaches are also used for improving the prediction accuracy of NN-based prediction model. In [93], authors retrained the NARNN prediction model using newly measured driving cycle data at each moving window step, and thus provided a more accurate prediction of driving cycles. Based on the predicted driving cycles, the proposed PEMS can achieve an optimized energy management result as offline DP method. In [42], a hybrid optimization based on GA and PSO was utilized to search for the optimal value of initial weights and thresholds for BPNN in order to improve the prediction accuracy. Moreover, traffic flow speed, even weather and workday/holiday conditions are also taken into consideration for training the multi-source power demand BPNN prediction models.

\subsubsection{Approaches to improve prediction robustness}

Online TPM updating algorithms are explored by many researchers for improving the robustness and accuracy of conventional Markov models. Authors in [26] used this method to update one column of TPMs of driver behavior prediction model at each time instant to make it fitting better with real driving conditions. From simulation results, it can be seen that the overall prediction accuracy improvements brought by updating algorithms for two real world driving cycles, where the first trace showed smooth accelerations and the second trace showed the steep accelerations, are $1.3 \%$ and $13.4 \%$, respectively. The reason behind big discrepancy between two different improvements is that the initial TPMs of first trace is already representative of its driving patterns, whereas in the case of more varied driving cycles the improvements of the updating algorithm are significant.

Similarly, in [51], based on the nearest-neighborhood method, an online TPM updating algorithm is implemented for the 3-D Markov chain driver model. Inspired by [52], authors in [64], two Markov velocity predictors based on Nearest Neighbor approach and Fuzzy Encoding approach are proposed. And the Reinforcement learning algorithm is utilized for learning transition probability of power demand. From the simulation results, it is noted that fuzzy encoding approach could bring down the prediction errors by $53.7 \%$ (one-step ahead) and 40.3\% (10-step ahead) compared with Nearest Neighbor based approaches. Combining with the recursive TPM online updating algorithm [72] and the Fuzzy encoding approach, better performances of PEMSs can be achieved by a bi-level EMS for hybrid tracked vehicles [73], where the future power demand can be predicted and fused into real-time control strategy computation. 
For practical applications, $100 \%$ accuracy of prediction is impossible to reach. In that case, PEMSs should not fully rely on the prediction, that is to say, when the prediction is recognized as inaccurate or invalid, backup EMSs should be used for energy distribution. In [62], a dynamic-neighborhood PSO (DPSO) based PEMS is proposed for reducing the fuel consumption of a PHEV. After that, an online correction algorithm is proposed to evaluate the prediction results by fuzzy logic controller. If prediction result are recognized as inaccurate, the backup Rule-based EMS will take the place of DPSO based PEMS to manage the power flow among the energy sources, which reduce the negative effects of prediction errors. From simulation results, it can be seen the deviations caused by prediction errors could be reduced by $32.39 \%$. Similarly, in [88], in order to cope with the false prediction of SoC reference value of PHEVs, online Distance Until Charging (DUC) algorithm was implemented to judge 1) whether "false prediction" events happen or not; 2) if there is "false prediction" , the reference curve of SoC will return to original statistical DUC distribution and activate the backup offline rule based EMS; 3) if there is no "false prediction", original real-time PEMS can still be effective. As a result, the proposed PEMS combined with DUC algorithm saved $4.64 \%$ more fuel consumption than rule based EMSs, which is slightly less than the results of DP based approaches, $5.89 \%$.

Based on the above analysis, approaches explored by researchers to cope with the mis-predictions are summarized in Table IV and corresponding references are listed after each approach.

\begin{tabular}{|c|c|c|}
\hline Classification & Sub-classification & Ref. \\
\hline \multirow[b]{2}{*}{$\begin{array}{l}\text { Reduce future } \\
\text { uncertainty }\end{array}$} & $\begin{array}{c}\text { Expand one-order Markov } \\
\text { Model to High-order Markov } \\
\text { Model }\end{array}$ & {$[23],[67],[69]$} \\
\hline & $\begin{array}{l}\text { Integrating Route-based } \\
\text { information into Prediction } \\
\text { framework }\end{array}$ & $\begin{array}{c}10],[11],[33]- \\
{[35],[56]-[61],} \\
{[71],[74],[75],} \\
{[77]-[79],[87],} \\
{[97],[98],[112],} \\
{[113],[116]}\end{array}$ \\
\hline \multirow{2}{*}{$\begin{array}{c}\text { Self-learning/Adaptive } \\
\text { Mechanism }\end{array}$} & $\begin{array}{c}\text { Adaptive/Self-learning Markov } \\
\text { approaches }\end{array}$ & $\begin{array}{c}{[51],[52],[64]} \\
{[72],[73]}\end{array}$ \\
\hline & Moving window Approaches & [93] \\
\hline \multirow{3}{*}{$\begin{array}{l}\text { Variable prediction } \\
\text { horizon }\end{array}$} & $\begin{array}{l}\text { Prediction with variable } \\
\text { horizon }\end{array}$ & $\begin{array}{c}{[19],[21],[49],} \\
{[50]}\end{array}$ \\
\hline & Multi-scale single step method & {$[19],[20],[50]$} \\
\hline & Online reconstruction of states & [49] \\
\hline $\begin{array}{l}\text { Reduce the dependency } \\
\text { on Predictions }\end{array}$ & $\begin{array}{l}\text { Backup strategy coping with } \\
\text { mis-predictions }\end{array}$ & {$[62]$} \\
\hline $\begin{array}{l}\text { Optimal tuned } \\
\text { parameters }\end{array}$ & $\begin{array}{l}\text { GA/PSO based parameter } \\
\text { optimization method }\end{array}$ & {$[69]$} \\
\hline
\end{tabular}

\section{OUTLOOK OF FDCS PREDICTION}

In the following part, a brief analysis about the outlook of driving prediction technique is conducted from practical application perspective.

\subsection{Multiple prediction algorithms integration}

To maximize the performances of each prediction method, suitable application scenarios should be carefully selected before prediction. However, practical application scenarios are usually combined with characteristics from many types of driving patterns. In this case, prediction performance will be poor if only one prediction method is used, because a single algorithm cannot always fit in well with every driving scenario. Consequently, in the future researches, multiple types of prediction methods should be utilized to make the results more credible in compound driving scenarios. For example, a novel velocity predicted method based on Wavelet transform (WT) and RBF-NN is proposed by Ningyuan G. et al [117] for the 
PEMS for PHEVs. Simulation results showed that the combined algorithm of 1-RBF-NN and WT achieved the accuracy improvement by $7.31 \%$ than the benchmarks. In [64], [72], [73], the combination of Reinforcement learning (RL) algorithm and Markov Chain was used to predict the power demand or vehicle velocity. And its robustness is verified by real world test data. In [118], a real-time PEMS was proposed combining with a Multi-step Markov velocity predictor and a RBF-NN DPR algorithm, whose prediction root mean square error (RMSE) was 1.2411 and brought corresponding fuel economy improvements (FEI) by $16.3 \%, 12.7 \%$ and $9.1 \%$ over three different driving cycles. It can be foreseen that in future researches, various prediction methods should be used together to compensate for shortcomings of each other, which will lead to better prediction performances.

\subsection{Online correction and update technique}

Conventional prediction models are fixed because most of them are generated from the stationary experimental database and there is no self-learning mechanism allowing them to acquire knowledge from external changes in real time. The discrepancy between stationary prediction results and actual values will become larger if there are not certain accuracycompensation measures, which will eventually lead to the bad performance on corresponding PEMSs. Online-correction part should be embedded into the EMSs framework, which adjusts the current prediction value based on the error of the prediction value by feedback mechanism.

Under such conditions, authors in [26] proposed a PEMS for driver-aware vehicles based on stochastic MPC with learning property. The framework combined with a self-learning
Markov driver behavior prediction model and a scenario-based decision tree approach for stochastic optimization. The selflearning Markov chain in this framework can "learn" the characteristic from newly-measured experimental data and reconfigure its TPMs to fit in well with changes in driver's behaviors. In [52], a generalized fuzzy coding based Markov model was proposed for real-time prediction and a real time recursive algorithm for learning transition probability from measured signal data is derived. Compared with interval encoding Markov chain, this technique can encode input data simultaneously into different states with a series of membership functions [64], [72], [73], which will result in higher prediction accuracy. Due to the requirements of real-time applications, online updating approaches and their applications for future driving conditions (FDCs) prediction will be a research hotspot in the future.

\subsection{Computation burden}

Computation burden is a key factor that influences the real time performance of prediction algorithms. For example, the prediction accuracy is improved when expanding traditional “one-order" Markov model into "high-order" Markov model [23]. However, as a side effect of that, the corresponding computation burden increases exponentially. Besides, the additional adaptive, self-learning process, the variable length of prediction horizon and the backup strategy will also bring extra computation time. In order to cope with such situations, measures like using simplified models [89] or improved high speed solvers [26], [65], [119]-[121] (e.g. fast QP solver [122], [123]) were explored by researchers. Therefore, for practical applications, tradeoffs should be made among the prediction 
accuracy and computation efficiency. Besides, advanced onboard processors or even cloud servers should be utilized to fit in with the rapid-growing computation requirements.

\subsection{Driver model and driving style recognition}

Different drivers' styles will lead to different driving actions (accelerating, decelerating, gear selecting etc.) in same driving scenarios. As a matter of fact, drivers' behaviors (driving habits) are always regarded as stochastic disturbances [26], [124], [125] of prediction models, which will have significant impacts on prediction accuracy and corresponding PEMSs performances. For example, in [126], three different types, including mild, normal and aggressive are explored in simulation with various drivers' models. The case study showed that in Central Business Districts (CBD), aggressive drivers consumed nearly $60 \%$ more fuel than that of normal drivers, while the mild drivers tended to be fuel efficient at the expense of the relative slow responses to required commands. Consequently, advanced drivers' driving style recognition techniques are therefore explored by many researchers in understanding drivers' future driving intentions to better control the power flow among different energy sources [36] and increase driving safety. A rapid driving style pattern-recognition approach was established by k-means clustering-based SVM [127], from the results of second groups in online case study, the proposed method improved the recognition accuracy by $9.78 \%$ for aggressive drivers and $17.06 \%$ for moderate drivers compared to conventional SVM classifier. In addition, total calculation time was greatly reduced from 740.18 s to $169.35 \mathrm{~s}$. The recognized driving styles can be used to choose different predesigned control strategies to improve FE as well as drivability for HEVs/PHEVs. Similarly, in [128], authors proposed a driving style recognition approach based on fuzzy logic. Four types of driving styles including below normal, normal, aggressive and very aggressive were the possible output of proposed recognizer. Benefiting from information from 2-axis accelerometer embedded in GPS, the high-accuracy recognition results can be obtained in real time with short computation expenses. Such recognition results are always used in traffic safety filed, like adaptive cruise control or forward collision warning (FCW). However, corresponding researches combining EMSs/PEMSs with drivers' driving styles to improve overall FE are rarely seen in current publications, which may be further investigated in the future.

\subsection{Multi-source information integration}

More driving related data is available with the rapid development of GPS, ITS, V2V and advanced sensor technologies. Useful prediction-assisting information like terrain profile [79], [87], [98], distance to next charging station [28], [88], distribution of traffic signal [33], [35], [58], traffic congestion level [61], [69] and the unexpected movements of preceding vehicles [60], [90] are integrated into conventional prediction models to enhance their performance in existing researches. In future researches, attentions should be paid to predict FDCs in a larger space scale and a longer timescale [5].

Researchers are eager to create a fully-connected platform, which includes the V2V network [129], vehicle-to-home network [130], vehicle-to-ITS network [131] and vehicle-togrid (V2G) network [132], to share and exchange driving related information for high precision prediction and intelligent PEMSs. For instance, in such intelligent connected 
transportation framework, a single vehicle can be regarded as the basic element of whole traffic flow. Benefiting the systematical information from each connected part, the final PEMSs can be implemented to adjust the dispatch of whole traffic flow to figure out a way of improving FE, expanding the lifetime of expensive energy-providing devices etc. [133]. Especially, for PEHVs, wireless power transfer technique and the connections among the smart house, micro grids and vehicles bring more flexibility in coordinating energy utilization during both driving and parking process [130]. Even the FEI of single vehicle should not be merely concentrated on a specific driving tasks in one or two day, instead, how to obtain long-term, e.g. one year or more, FEI on average levels and corresponding performances combined with energy sources sizing and degradation issues will be more attractive in future researches.

\section{CONCLUSION}

Future driving conditions (FDCs) prediction is the basis of corresponding predictive energy management strategies (PEMSs) for HEVs/PHEVs and the quality of prediction results greatly affected the performance of PEMSs. This paper presents a comprehensive study on driving prediction techniques (DPTs). Through detailed review and comparisons of FDCs prediction algorithms used for DPTs, it is clear that each of them has own pros and cons, which makes them suitable for different application scenarios. For practical applications, it is significant to utilize the combination of different types of prediction methods together to fit in with various changeable external environments. Moreover, proper tradeoffs should be made between computational burden and prediction accuracy to satisfy the real-time requirements. Based on the analysis of the accuracy-affecting factors, the mechanism how these factors affect the prediction accuracy is clear and then corresponding approaches for improving prediction accuracy and robustness are discussed and summarized. Finally, the outlook of DPTs is conducted to give the developing trends of this technique.

In conclusion, major contributions of the presented survey are summarized as follows:

- A comprehensive review on DPTs conducted in this paper could provide useful information on recent progress in the field of PEMS for PHEVs;

- Comparative studies and scenario-based analyses on various prediction approaches could be used as general selection criteria according to different application backgrounds;

- Sources of prediction errors and corresponding strategies to cope with mis-predictions were thoroughly discussed to provide potential ways of improving accuracy and robustness of prediction approaches.

Additionally, based on the analysis of missing points in current researches, several potential directions of future researches on DPTs are indicated as follows:

- In order to adapt for changeable driving conditions, it is highly recommended to explore multi-algorithm based prediction frameworks and introduce online update mechanisms for learning novel characteristics from external driving environments;

- Advanced techniques on intelligent driver models including driving behavior prediction and driving styles 
recognitions, should be further investigated to explore the quantitative relationships between driver models and fuel economy performances;

- Corresponding performances on PEMSs could be highly improved, since multi-source information from modern telematics systems, including GPS, GIS and ITS, could be combined for reducing the uncertainty of future driving conditions to further improve prediction accuracy;

- $\quad$ Benefiting from rapid development of advanced vehicular technologies, such as $\mathrm{V} 2 \mathrm{~V}, \mathrm{~V} 2 \mathrm{G}$, Internet of Vehicles (IoV) and wireless power transfer, novel possibility of PEMS for HEVs/PHEVs should be explored on larger space scales and longer time scales.

Original insights on bottleneck of current researches and potential future developing tendency on Driving Prediction Techniques proposed in this section could provide prospective researchers with novel inspirations and general guidelines for their future works.

\section{REFERENCES}

[1] R. Wang and S. M. Lukic, "Review of driving conditions prediction and driving style recognition based control algorithms for hybrid electric vehicles," 2011 IEEE Veh. Power Propuls. Conf. VPPC 2011, 2011.

[2] P. Zhang, F. Yan, and C. Du, "A comprehensive analysis of energy management strategies for hybrid electric vehicles based on bibliometrics," Renew. Sustain. Energy Rev., vol. 48, no. 205, pp. 88-104, 2015.

[3] Y. Huang, H. Wang, A. Khajepour, H. He, and J. Ji, "Model predictive control power management strategies for HEVs: A review," J. Power Sources, vol. 341, pp. 91-106, 2017.

[4] S. G. Wirasingha and A. Emadi, "Classification and Review of Control Strategies for Plug-In Hybrid Electric Vehicles,” Veh. Technol. IEEE Trans., vol. 60, no. 1, pp. 111-122, 2011. C. Marina Martinez, X. Hu, D. Cao, E. Velenis, B. Gao, and M. Wellers, "Energy Management in Plug-in Hybrid Electric Vehicles: Recent Progress and a Connected Vehicles Perspective," IEEE Trans. Veh. Technol., vol. PP, no. 99, pp. 1-1, 2016.
Modeling and Optimization. Springer, 2005.

[7] M. Ehsani, "Design and Control Methodology of Plug-in Hybrid Electric Vehicles," IEEE Trans. Ind. Electron., vol. 57, no. 2, pp. 633-640, 2010.

J. Gonder and T. Markel, "Energy Management Strategies for PlugIn Hybrid Electric Vehicles Distance," SAE World Congr., pp. 1-5, 2007.

[9] M. Zhang, Y. Yang, and C. C. Mi, "Analytical approach for the power management of blended-mode plug-in hybrid electric vehicles," IEEE Trans. Veh. Technol., vol. 61, no. 4, pp. 15541566, 2012.

[10] G. Qiuming, L. Yaoyu, and P. Zhong-Ren, "Trip based optimal power management of plug-in hybrid electric vehicles using gaskinetic traffic flow model," IEEE Trans. Veh. Technol., vol. 57, no. 6, pp. 3225-3230, 2008.

[11] C. Zhang and A. Vahidi, "Route preview in energy management of plug-in hybrid vehicles," IEEE Trans. Control Syst. Technol., vol. 20, no. 2, pp. 546-553, 2012.

[12] P. Tulpule, V. Marano, and G. Rizzoni, "Effects of different PHEV control strategies on vehicle performance," Proc. Am. Control Conf., pp. 3950-3955, 2009.

[13] C. Sun, S. J. Moura, X. Hu, J. K. Hedrick, and F. Sun, "Dynamic Traffic Feedback Data Enabled Energy Management in Plug-in Hybrid Electric Vehicles," IEEE Trans. Control Syst. Technol., vol. 23, no. 3, pp. 1075-1086, 2015.

[14] H. He, R. Xiong, K. Zhao, and Z. Liu, "Energy management strategy research on a hybrid power system by hardware-in-loop experiments," Appl. Energy, vol. 112, pp. 1311-1317, 2013.

[15] X. Wang, H. He, F. Sun, and J. Zhang, "Application study on the dynamic programming algorithm for energy management of plug-in hybrid electric vehicles," Energies, vol. 8, no. 4, pp. 3225-3244, 2015.

[16] S. Kermani, S. Delprat, T. M. Guerra, R. Trigui, and B. Jeanneret, "Predictive energy management for hybrid vehicle," Control Eng. Pract., vol. 20, no. 4, pp. 408-420, 2012.

[17] T. S. Kim, C. Manzie, and R. Sharma, "Model predictive control of velocity and torque split in a parallel hybrid vehicle," Conf. Proc. IEEE Int. Conf. Syst. Man Cybern., no. October 2009, pp. 20142019, 2009.

[18] H. Banvait, S. Member, and J. Hu, "Energy Management Control of Plug-in Hybrid Electric Vehicle using Hybrid Dynamical Systems Set of discrete inputs," IEEE Trans. Intell. Transp. Syst., vol. XX, no. X, pp. 1-13.

[19] S. Xie, H. He, and J. Peng, “An energy management strategy based on stochastic model predictive control for plug-in hybrid electric buses," Appl. Energy, vol. 196, pp. 279-288, 2017.

[20] G. Li, J. Zhang, and H. He, "Battery SOC constraint comparison for predictive energy management of plug-in hybrid electric bus," Appl. Energy, vol. 194, pp. 578-587, 2017.

[21] J. Cao, J. Peng, and H. He, "Research on Model Prediction Energy Management Strategy with Variable Horizon," Energy Procedia, vol. 105, pp. 3565-3570, 2017.

[22] H. Li, A. Ravey, A. N'Diaye, and A. Djerdir, “A novel equivalent consumption minimization strategy for hybrid electric vehicle 
powered by fuel cell, battery and supercapacitor," J. Power Sources, vol. 395, no. February, pp. 262-270, 2018.

C. Sun, X. Hu, S. J. Moura, and F. Sun, "Velocity Predictors for Predictive Energy Management in Hybrid Electric Vehicles," IEEE Trans. Control Syst. Technol., vol. 23, no. 3, pp. 1197-1204, 2015.

I. Arsie, M. Graziosi, C. Pianese, G. Rizzo, and M. Sorrentino,

"Control strategy optimization for hybrid electric vehicles via provisional load estimate," Rev. Automot. Eng., 2005.

J. P. J. Park, Z. C. Z. Chen, and Y. L. Murphey, "Intelligent vehicle power management through neural learning," Neural Networks IJCNN 2010 Int. Jt. Conf., pp. 18-23, 2010.

[26] S. Di Cairano, D. Bernardini, A. Bemporad, and I. V. Kolmanovsky, "Stochastic MPC with learning for driver-predictive vehicle control and its application to HEV energy management," IEEE Trans. Control Syst. Technol., vol. 22, no. 3, pp. 1018-1031, 2014.

[27] G. Ripaccioli, D. Bernardini, S. Di Cairano, A. Bemporad, and I. V Kolmanovsky, "A stochastic model predictive control approach for series hybrid electric vehicle power management," in Proceedings of the 2010 American Control Conference, 2010, pp. 5844-5849.

[28] P. Naghshtabrizi, J. Kristinsson, H. Yu, and R. McGee, "Distance Until Charge prediction and fuel economy impact for Plug-in Hybrid Vehicles," Proc. 2011 Am. Control Conf., pp. 2739-2744, 2011.

[29] M. Montazeri-Gh and Z. Pourbafarani, "Near-optimal SOC trajectory for traffic-based adaptive PHEV control strategy," IEEE Trans. Veh. Technol., vol. 66, no. 11, pp. 9753-9760, 2017.

[30] J. Wang, Y. Huang, H. Xie, and G. Tian, "Driving Pattern Prediction Model for Hybrid Electric Buses Based on Real-World Driving Data," EVS28 Int. Electr. Veh. Symp. Exhib., pp. 1-9, 2015.

[31] S. Zhang and R. Xiong, "Adaptive energy management of a plug-in hybrid electric vehicle based on driving pattern recognition and dynamic programming," Appl. Energy, vol. 155, pp. 68-78, 2015.

[32] W. Song, X. Zhang, Y. Tian, X. Zhang, and J. Song, "The research on vehicle driving pattern characteristic parameters search algorithm based on parallel computing," Proc. 33rd Chinese Control Conf. CCC 2014, pp. 147-150, 2014.

[33] B. Asadi and A. Vahidi, "Predictive cruise control: Utilizing upcoming traffic signal information for improving fuel economy and reducing trip time," IEEE Trans. Control Syst. Technol., vol. 19, no. 3, pp. 707-714, 2011.

[34] K. R. Bouwman, T. H. Pham, S. Wilkins, and T. Hofman, "Predictive Energy Management Strategy Including Traffic Flow Data for Hybrid Electric Vehicles," IFAC-PapersOnLine, vol. 50, no. 1, pp. 10046-10051, 2017.

[35] G. Mahler and A. Vahidi, "An optimal velocity-planning scheme for vehicle energy efficiency through probabilistic prediction of trafficsignal timing," IEEE Trans. Intell. Transp. Syst., vol. 15, no. 6, pp. 2516-2523, 2014.

[36] C. M. Martinez, M. Heucke, F. Y. Wang, B. Gao, and D. Cao, "Driving Style Recognition for Intelligent Vehicle Control and Advanced Driver Assistance: A Survey,” IEEE Trans. Intell. Transp. Syst., vol. PP, no. 99, pp. 1-11, 2017.

[37] D. A. Johnson and M. M. Trivedi, "Driving Style Recognition Using a Smartphone As a Sensor Platform -

Johnson_ITSC2011.pdf," Academia.Edu, pp. 1609-1615, 2011.
[38] G. Lenaers, "Real Life CO2 Emission and Consumption of Four Car Powertrain Technologies Related to Driving Behaviour and Road Type," SAE Tech. Pap. Ser., 2009.

[39] C. Lin, S. Jeon, H. Peng, and J. M. O. O. Lee, "Driving Pattern Recognition for Control of Hybrid Electric Trucks."

[40] H. Yu, F. Tseng, and R. McGee, "Driving pattern identification for EV range estimation," 2012 IEEE Int. Electr. Veh. Conf. IEVC 2012, 2012.

[41] C. Sun, H. He, and F. Sun, "The Role of Velocity Forecasting in Adaptive-ECMS for Hybrid Electric Vehicles," Energy Procedia, vol. 75, pp. 1907-1912, 2015.

[42] J. Liu, Y. Chen, J. Zhan, and F. Shang, “An On-line Energy Management Strategy based on Trip Condition Prediction for Commuter Plug-in Hybrid Electric Vehicles," IEEE Trans. Veh. Technol., vol. 9545, no. c, pp. 1-1, 2018.

[43] J. Lemieux and Y. Ma, "Vehicle Speed Prediction Using Deep Learning,” 2015 IEEE Veh. Power Propuls. Conf., pp. 1-5, 2015.

[44] C. Xiang, F. Ding, W. Wang, and W. He, "Energy management of a dual-mode power-split hybrid electric vehicle based on velocity prediction and nonlinear model predictive control," Appl. Energy, vol. 189, pp. 640-653, 2017.

[45] L. Liu, C. Huang, B. Lu, S. Shi, Y. Zhang, and J. Cheng, "Study on the design method of time-variant driving cycles for EV based on Markov Process," 2012 IEEE Veh. Power Propuls. Conf. VPPC 2012, pp. 1277-1281, 2012.

[46] S. Xie, J. Peng, and H. He, "Plug-In Hybrid Electric Bus Energy Management Based on Stochastic Model Predictive Control," Energy Procedia, vol. 105, no. 10, pp. 2672-2677, 2017.

[47] S. Shi, N. Lin, Y. Zhang, J. Cheng, C. Huang, L. Liu, and B. Lu, "Research on Markov property analysis of driving cycles and its application," Transp. Res. Part D Transp. Environ., vol. 47, pp. 171-181, 2016.

[48] D. Huang, H. Xie, H. Ma, and Q. Sun, "Driving cycle prediction model based on bus route features," Transp. Res. Part D Transp. Environ., vol. 54, pp. 99-113, 2017.

[49] H. He, J. Cao, and J. Peng, "Online Prediction with Variable Horizon for Vehicle's Future Driving-Cycle," Energy Procedia, vol. 105, pp. 2348-2353, 2017.

[50] Y. Li, J. Peng, H. He, and S. Xie, "The Study on Multi-scale Prediction of Future Driving Cycle Based on Markov Chain," Energy Procedia, vol. 105, pp. 3219-3224, 2017.

[51] Y. Zou, Z. Kong, T. Liu, and D. Liu, “A real-time Markov chain driver model for tracked vehicles and its validation: Its adaptability via stochastic dynamic programming," IEEE Trans. Veh. Technol., vol. 66, no. 5, pp. 3571-3582, 2017.

[52] D. P. Filev and I. Kolmanovsky, "Generalized Markov models for real-time modeling of continuous systems," IEEE Trans. Fuzzy Syst., vol. 22, no. 4, pp. 983-998, 2014.

[53] S. Zhang, R. Xiong, and F. Sun, "Model predictive control for power management in a plug-in hybrid electric vehicle with a hybrid energy storage system," Appl. Energy, vol. 185, pp. 1654-1662, 2017.

[54] H. A. Borhan and A. Vahidi, "Model predictive control of a powersplit Hybrid Electric Vehicle with combined battery and 
ultracapacitor energy storage," Proc. 2010 Am. Control Conf., pp. 5031-5036, 2010.

H. Borhan, S. Member, A. Vahidi, A. M. Phillips, M. L. Kuang, I. V Kolmanovsky, and S. Di Cairano, "MPC-Based Energy

Management of a Power-Split Hybrid Electric Vehicle," vol. 20, no. 3, pp. 593-603, 2012.

[56] D. F. Opila, "Uncertain route, destination, and traffic predictions in energy management for hybrid, plug-in, and fuel-cell vehicles," Proc. Am. Control Conf., vol. 2016-July, pp. 1685-1692, 2016.

H. Hongwen, G. Jinquan, P. Jiankun, T. Huachun, and S. Chao, "Real-time global driving cycle construction and the application to economy driving pro system in plug-in hybrid electric vehicles," Energy, 2018.

[58] L. Qiu, L. Qian, H. Zomorodi, and P. Pisu, "Global optimal energy management control strategies for connected four-wheel-drive hybrid electric vehicles," IET Intell. Transp. Syst., vol. 11, no. 5, pp. 264-272, 2017.

[59] X. Zeng and J. Wang, "A Parallel Hybrid Electric Vehicle Energy Management Strategy Using Stochastic Model Predictive Control with Road Grade Preview," IEEE Trans. Control Syst. Technol., vol. 23, no. 6, pp. 2416-2423, 2015.

[60] M. A. S. Kamal, M. Mukai, J. Murata, and T. Kawabe, "Model predictive control of vehicles on urban roads for improved fuel economy," IEEE Trans. Control Syst. Technol., vol. 21, no. 3, pp. 831-841, 2013.

[61] L. Guo, B. Gao, Y. Gao, and H. Chen, "Optimal Energy Management for HEVs in Eco-Driving Applications Using Bi-Level MPC," IEEE Trans. Intell. Transp. Syst., vol. 18, no. 8, pp. 21532162, 2017.

[62] Z. Chen, R. Xiong, C. Wang, and J. Cao, “An on-line predictive energy management strategy for plug-in hybrid electric vehicles to counter the uncertain prediction of the driving cycle," Appl. Energy, vol. 185, pp. 1663-1672, 2017.

[63] F. A. Bender, H. Uzuner, and O. Sawodny, An adaptive driver model for driving cycle prediction in the intelligent truck, vol. 19, no. 3. IFAC, 2014.

[64] T. Liu, X. Hu, S. E. Li, and D. Cao, "Reinforcement Learning Optimized Look-Ahead Energy Management of a Parallel Hybrid Electric Vehicle," IEEE/ASME Trans. Mechatronics, vol. 22, no. 4, pp. 1497-1507, 2017.

[65] Z. Chen, Y. Wu, N. Guo, J. Shen, and R. Xiao, "Energy management for plug-in hybrid electric vehicles based on quadratic programming with optimized engine on-off sequence," IECON 2017 - 43rd Annu. Conf. IEEE Ind. Electron. Soc., pp. 7134-7139, 2017.

[66] Z. Chen, L. Li, B. Yan, C. Yang, C. Marina Martinez, and D. Cao, "Multimode Energy Management for Plug-In Hybrid Electric Buses Based on Driving Cycles Prediction," IEEE Trans. Intell. Transp. Syst., vol. 17, no. 10, pp. 2811-2821, 2016.

[67] J. Zhang, H. He, and X. Wang, "Model Predictive Control Based Energy Management Strategy for a Plug-In Hybrid Electric Vehicle," in Icmeis, 2015, vol. 1, no. Icmeis, pp. 875-879.

[68] F. Tianheng, Y. Lin, G. Qing, H. Yanqing, Y. Ting, and Y. Bin, “A supervisory control strategy for plug-in hybrid electric vehicles based on energy demand prediction and route preview," IEEE Trans. Veh. Technol., vol. 64, no. 5, pp. 1691-1700, 2015.
[69] T. Li, H. Liu, and D. Ding, "Predictive energy management of fuel cell supercapacitor hybrid construction equipment," Energy, vol. 149, pp. 718-729, 2018.

[70] S. J. Moura, H. K. Fathy, D. S. Callaway, and J. L. Stein, “A Stochastic Optimal Control Approach for Power Management in Plug-In Hybrid Electric Vehicles," Control Syst. Technol. IEEE Trans., vol. 19, no. 3, pp. 545-555, 2011.

[71] Y. Bin, Y. Li, Q. Gong, and Z. R. Peng, "Multi-information integrated trip specific optimal power management for plug-in hybrid electric vehicles," Proc. Am. Control Conf., pp. 4607-4612, 2009.

[72] Y. Zou, T. Liu, D. Liu, and F. Sun, "Reinforcement learning-based real-time energy management for a hybrid tracked vehicle," Appl. Energy, vol. 171, pp. 372-382, 2016.

[73] T. Liu and X. Hu, “A Bi-Level Control for Energy Efficiency Improvement of a Hybrid Tracked Vehicle," IEEE Trans. Ind. Informatics, vol. 14, no. 4, pp. 1616-1625, 2018.

[74] S. Kelouwani, N. Henao, K. Agbossou, Y. Dube, and L. Boulon, "Two-layer energy-management architecture for a fuel cell HEV using road trip information," IEEE Trans. Veh. Technol., vol. 61, no. 9, pp. 3851-3864, 2012.

[75] Y. Ma and J. Wang, "Integrated power management and aftertreatment system control for hybrid electric vehicles with road grade preview," IEEE Trans. Veh. Technol., vol. 66, no. 12, pp. 10935-10945, 2017.

[76] N. Guo, J. Shen, R. Xiao, W. Yan, and Z. Chen, "Energy Management for Plug-in Hybrid Electric Vehicles Considering Optimal Engine ON/ OFF Control and Fast State-of-Charge Trajectory Planning,” Energy, vol. 163, pp. 457-474, 2018.

[77] S. Cordiner, M. Galeotti, V. Mulone, M. Nobile, and V. Rocco, "Trip-based SOC management for a plugin hybrid electric vehicle," Appl. Energy, vol. 164, pp. 891-905, 2016.

[78] B. Geng, J. K. Mills, and D. Sun, "Two-stage energy management control of fuel cell plug-in hybrid electric vehicles considering fuel cell longevity," IEEE Trans. Veh. Technol., vol. 61, no. 2, pp. 498508, 2012.

[79] Y. Zhao, Y. Cai, and Q. Song, "Energy Control of Plug-In Hybrid Electric Vehicles," IEEE/CAA J. Autom. Sin., pp. 1-8.

[80] Q. Zhang, W. Deng, and G. Li, "Stochastic Control of Predictive Power Management for Battery/Supercapacitor Hybrid Energy Storage Systems of Electric Vehicles," IEEE Trans. Ind. Informatics, vol. 3203, no. c, pp. 1-8, 2017.

[81] T. Yi, Z. Xin, Z. Liang, and Z. Xinn, "Intelligent energy management based on driving cycle identification using fuzzy neural network," Isc. 2009 - 2009 Int. Symp. Comput. Intell. Des., vol. 2, no. Iwisa, pp. 501-504, 2009.

[82] R. Langari and J.-S. W. J.-S. Won, "Intelligent energy management agent for a parallel hybrid vehicle-part I: system architecture and design of the driving situation identification process," IEEE Trans. Veh. Technol., vol. 54, no. 3, pp. 925-934, 2005.

[83] E. Ericsson, "Independent driving pattern factors and their influence on fuel-use and exhaust emission factors," Transp. Res. Part D Transp. Environ., vol. 6, no. 5, pp. 325-345, 2001.

[84] Q. Zhang and W. Deng, "An adaptive energy management system for electric vehicles based on driving cycle identification and 
wavelet transform," Energies, vol. 9, no. 5, 2016.

[85]

F. Soriano, M. Moreno-Eguilaz, and J. ??lvarez-Fl??rez, "Drive Cycle Identification and Energy Demand Estimation for RefuseCollecting Vehicles," IEEE Trans. Veh. Technol., vol. 64, no. 11, pp. 4965-4973, 2015.

[86] Y. L. Murphey, Z. H. Chen, L. Kiliaris, J. Park, M. Kuang, A. Masrur, and A. Phillips, "Neural learning of driving environment prediction for vehicle power management," Proc. Int. Jt. Conf. Neural Networks, pp. 3755-3761, 2008.

[87] C. Zhang, A. Vahidi, P. Pisu, L. Xiaopeng, and K. Tennant, "Role of Terrain Preview in Energy Management of Hybrid Electric Vehicles," Veh. Technol. IEEE Trans., vol. 59, no. 3, pp. 11391147, 2010.

[88] J. Lohrer, M. Förth, and M. Lienkamp, "A Data-Driven Predictive Energy Management Strategy for Plug-in Hybrid Vehicles,” pp. 297-304, 2017.

[89] A. Ravey, R. Wang, S. Lukic, and A. Miraoui, "Distance estimation algorithm for plug-in hybrid electric vehicle control strategy," in 2013 IEEE Transportation Electrification Conference and Expo (ITEC), 2013, pp. 1-4.

[90] S. Zhang, Y. Luo, K. Li, and J. Wang, "Predictive Energy Management Strategy for Fully Electric Vehicles based on Hybrid Model Predictive Control," 2017 Am. Control Conf., pp. 36253630, 2017.

[91] Z. Chen, L. Kiliaris, Y. L. Murphey, and M. A. Masrur, "Intelligent power management in SHEV based on roadway type and traffic congestion levels," 5th IEEE Veh. Power Propuls. Conf. VPPC '09, pp. 915-920, 2009.

[92] Y. Li, H. He, J. Peng, and H. Zhang, "Power Management for a Plug-in Hybrid Electric Vehicle Based on Reinforcement Learning with Continuous State and Action Spaces," Energy Procedia, vol. 142, pp. 2270-2275, 2017.

[93] D. Zhou, F. Gao, and A. Ravey, "Online Energy Management Strategy of Fuel Cell Hybrid Electric Vehicles Based on Time Series Prediction," 2017 IEEE Transp. Electrif. Conf. Expo, pp. 113-118, 2017.

[94] Y. Huang, H. Wang, A. Khajepour, H. He, and J. Ji, "Model predictive control power management strategies for HEVs: A review," J. Power Sources, vol. 341, pp. 91-106, 2017.

[95] M. Joševski and D. Abel, "Tube-based MPC for the energy management of hybrid electric vehicles with non-parametric driving profile prediction," in Proceedings of the American Control Conference, 2016, vol. 2016-July, pp. 623-630.

[96] H. A. . A. V. Borhan, "predictive energy management of a powersplit hybrid electric vehicle," Am. Control Conf., 2009.

[97] Z. Chen, C. C. Mi, J. Xu, X. Gong, and C. You, "Energy management for a power-split plug-in hybrid electric vehicle based on dynamic programming and neural networks," IEEE Trans. Veh. Technol., vol. 63, no. 4, pp. 1567-1580, 2014.

[98] C. Zhang and A. Vahidi, "Real-time optimal control of plug-in hybrid vehicles with trip preview," 2010 Am. Control Conf., pp. 6917-6922, 2010.

[99] J. Buerger, S. East, and M. Cannon, "Fast dual loop nonlinear receding horizon control for energy management in hybrid electric vehicles," Submitt. to IEEE Trans. Control Syst. Technol., pp. 1-11,
2017.

[100] H. He, J. Guo, and C. Sun, "Road Grade Prediction for Predictive Energy Management in Hybrid Electric Vehicles," Energy Procedia, vol. 105, pp. 2438-2444, 2017.

[101] V. Larsson, L. J. Mårdh, B. Egardt, and S. Karlsson, "Commuter Route Optimized Energy Management of Hybrid Electric Vehicles," vol. 15, no. 3, pp. 1-10, 2014.

[102] J. Brady and M. O'Mahony, "Development of a driving cycle to evaluate the energy economy of electric vehicles in urban areas," Appl. Energy, vol. 177, pp. 165-178, 2016.

[103] Z. Wei, J. Xu, and D. Halim, "HEV power management control strategy for urban driving," Appl. Energy, vol. 194, pp. 705-714, 2017.

[104] D. Zhou, A. Al-Durra, F. Gao, A. Ravey, I. Matraji, and M. Godoy Simões, "Online energy management strategy of fuel cell hybrid electric vehicles based on data fusion approach," J. Power Sources, vol. 366, pp. 278-291, 2017.

[105] W. Liu, H. He, and F. Sun, "Vehicle state estimation based on Minimum Model Error criterion combining with Extended Kalman Filter,” J. Franklin Inst., vol. 353, no. 4, pp. 834-856, 2015.

[106] G. Qiuming, L. Yaoyu, and P. Zhongren, "Power management of plug-in hybrid electric vehicles using neural network based trip modeling," Am. Control Conf. 2009, pp. 4601-4606, 2009.

[107] C. Sun, F. Sun, and H. He, "Investigating adaptive-ECMS with velocity forecast ability for hybrid electric vehicles," Appl. Energy, vol. 185, pp. 1644-1653, 2017.

[108] J. Wu, H. He, J. Peng, Y. Li, and Z. Li, “Continuous reinforcement learning of energy management with deep Q network for a power split hybrid electric bus," Appl. Energy, vol. 222, no. January, pp. 799-811, 2018.

[109] P. M. Muñoz, G. Correa, M. E. Gaudiano, and D. Fernández, "Energy management control design for fuel cell hybrid electric vehicles using neural networks," Int. J. Hydrogen Energy, vol. 42, no. 48, pp. 28932-28944, 2017.

[110] R. Xiong, J. Cao, and Q. Yu, "Reinforcement learning-based realtime power management for hybrid energy storage system in the plug-in hybrid electric vehicle," Appl. Energy, vol. 211, no. 5, pp. 538-548, 2018.

[111] L. Li, S. You, C. Yang, B. Yan, J. Song, and Z. Chen, "Drivingbehavior-aware stochastic model predictive control for plug-in hybrid electric buses,” Appl. Energy, vol. 162, pp. 868-879, 2016.

[112] P. Golchoubian and N. L. Azad, "Real-Time Nonlinear Model Predictive Control of a Battery-Supercapacitor Hybrid Energy Storage System in Electric Vehicles," IEEE Trans. Veh. Technol., vol. 66, no. 11, pp. 9678-9688, 2017.

[113] D. Karbowski, N. Kim, and A. Rousseau, "Route-based online energy management of a PHEV and sensitivity to trip prediction," 2014 IEEE Veh. Power Propuls. Conf. VPPC 2014, 2014.

[114] J. Eckstein, C. Lüke, F. Brunstein, P. Friedel, U. Köhler, and A. Trächtler, "A Novel Approach Using Model Predictive Control to Enhance the Range of Electric Vehicles," Procedia Technol., vol. 26, pp. 177-184, 2016.

[115] O. Gomozov, J. P. F. Trovao, X. Kestelyn, and M. R. Dubois, "Adaptive Energy Management System Based on a Real-Time 
Model Predictive Control with Nonuniform Sampling Time for Multiple Energy Storage Electric Vehicle," IEEE Trans. Veh. Technol., vol. 66, no. 7, pp. 5520-5530, 2017.

[116] Z. D. Asher, D. A. Baker, and T. H. Bradley, "Prediction Error Applied to Hybrid Electric Vehicle Optimal Fuel Economy," IEEE Trans. Control Syst. Technol., pp. 1-14, 2017.

[117] N. Guo, Z. Chen, Y. Wu, J. Shen, and R. Xiao, "A Novel Velocity Forecast Method for Improving Predictive Energy Management of Plug-in Hybrid Electric Vehicles," no. 2015, 2017.

[118] H. Liu, X. Li, W. Wang, L. Han, and C. Xiang, "Markov velocity predictor and radial basis function neural network-based real-time energy management strategy for plug-in hybrid electric vehicles," Energy, vol. 152, pp. 427-444, 2018.

[119] Z. Chen, C. C. Mi, R. Xiong, J. Xu, and C. You, "Energy management of a power-split plug-in hybrid electric vehicle based on genetic algorithm and quadratic programming," J. Power Sources, vol. 248, pp. 416-426, 2014.

[120] Z. Chen, C. C. Mi, B. Xia, and C. You, "Energy management of power-split plug-in hybrid electric vehicles based on simulated annealing and Pontryagin's minimum principle," J. Power Sources, vol. 272, pp. 160-168, 2014.

[121] Z. Chen, B. Xia, C. You, and C. C. Mi, "A novel energy management method for series plug-in hybrid electric vehicles," Appl. Energy, vol. 145, pp. 172-179, 2015.

[122] S. Richter, C. N. Jones, and M. Morari, "Computational complexity certification for real-time MPC with input constraints based on the fast gradient method," IEEE Trans. Automat. Contr., vol. 57, no. 6, pp. 1391-1403, 2012.

[123] Y. Pu, M. N. Zeilinger, and C. N. Jones, "Complexity certification of the fast alternating minimization algorithm for linear MPC," IEEE Trans. Automat. Contr., vol. 62, no. 2, pp. 888-893, 2017.

[124] M. Da Lio, A. Mazzalai, K. Gurney, and A. Saroldi, "Biologically

\section{Authors INFO:}

Yang Zhou (corresponding author) was born in Xi'an, China. $\mathrm{He}$ received the B.Eng. and M.S. degrees in electrical engineering from the Northwestern Polytechnical University (NWPU), Xi'an, China, in 2014 and 2017, respectively.

He is currently a Ph.D. student at FEMTO-ST (UMR CNRS 6174), Department of Energy, and FCLAB (FR CNRS 3539), University of Bourgogne Franche-Comte, UTBM, Belfort, France. His main research interests include the predictive energy management of fuel cell hybrid electric vehicles. Email: yang.zhou@utbm.fr.

Alexandre Ravey (S'10-M'12) received the M.S. and Ph.D. degrees in electrical engineering from the University of Technology of Belfort-Montbeliard (UTBM), Belfort, France, in 2009 and 2012, respectively.

Since 2013, he has been an Associate Professor with the UTBM. He is currently involved in Mobypost European project, which is aiming to build a hybrid electric vehicle based on fuel cell and batteries. His research interests include the energy management of electric and hybrid vehicles.
Guided Driver Modeling: the Stop Behavior of Human Car Drivers," IEEE Trans. Intell. Transp. Syst., pp. 1-16, 2017.

[125] W. Wang, "Evaluation of Lane Departure Correction Systems Using a Regenerative Stochastic Driver Model," IEEE Trans. Intell. Veh., vol. 2, no. 3, pp. 221-232, 2017.

[126] S. Zorrofi, S. Filizadeh, and P. Zanetel, "A simulation study of the impact of driving patterns and driver behavior on fuel economy of hybrid transit buses," 5th IEEE Veh. Power Propuls. Conf. VPPC '09, pp. 572-577, 2009.

[127] W. Wang and J. Xi, "A rapid pattern-recognition method for driving styles using clustering-based support vector machines," Proc. Am. Control Conf., vol. 2016-July, pp. 5270-5275, 2016.

[128] A. Aljaafreh, N. Alshabatat, and M. S. Najim Al-Din, "Driving style recognition using fuzzy logic," 2012 IEEE Int. Conf. Veh. Electron. Safety, ICVES 2012, pp. 460-463, 2012.

[129] B. HomChaudhuri, R. Lin, and P. Pisu, "Hierarchical control strategies for energy management of connected hybrid electric vehicles in urban roads," Transp. Res. Part C Emerg. Technol., vol. 62, pp. 70-86, 2016.

[130] Y. Mou, H. Xing, Z. Lin, and M. Fu, "Decentralized optimal demand-side management for PHEV charging in a smart grid," IEEE Trans. Smart Grid, vol. 6, no. 2, pp. 726-736, 2015.

[131] D. Jia, K. Lu, J. Wang, X. Zhang, and X. Shen, "A survey on platoon-based vehicular cyber-physical systems," IEEE Commun. Surv. Tutorials, vol. 18, no. 1, pp. 263-284, 2016.

[132] A. Mohamed, V. Salehi, T. Ma, and O. Mohammed, "Real-time energy management algorithm for plug-in hybrid electric vehicle charging parks involving sustainable energy," IEEE Trans. Sustain. Energy, vol. 5, no. 2, pp. 577-586, 2014.

[133] A. A. Malikopoulos, "Supervisory power management control algorithms for hybrid electric vehicles: A survey," IEEE Trans. Intell. Transp. Syst., vol. 15, no. 5, pp. 1869-1885, 2014.

\section{Email: alexandre.ravey@utbm.fr.}

Marie-Cécile Péra received the Master degree in electrical engineering from the Institut National Polytechnique de Grenoble, Grenoble, France, in 1990 and the Ph.D. degree in electrical engineering from the Institut National Polytechnique de Grenoble in 1993.

She works on energy management of hybrid electric power generation systems (fuel cells, PEMFC and SOFC, supercapacities, and batteries) and the diagnosis and prognostics of fuel cell systems. Since 2012, she has been the Deputy Director of the FEMTO-ST Institute (800 people) and a member of the FCLAB Research Federation. She is a Member of both the Scientific Council of the Department of Engineering and Systems Sciences, National Center for Scientific Research (CNRS), and of the French National Council of CNRS.

Email: marie-cecile.pera@univ-fcomte.fr. 